\title{
Diquarks and nucleons under strong magnetic fields in the NJL model
}

\author{
M. Coppola $\oplus^{1,2}$ D. Gomez Dumm, ${ }^{3}$ and N. N. Scoccola ${ }^{1,2}$ \\ ${ }^{1}$ CONICET, Rivadavia 1917, 1033 Buenos Aires, Argentina \\ ${ }^{2}$ Physics Department, Comisión Nacional de Energía Atómica, Avenue Libertador 8250, \\ 1429 Buenos Aires, Argentina \\ ${ }^{3}$ IFLP, CONICET-Departamento de Física, Facultad de Ciencias Exactas, \\ Universidad Nacional de La Plata, C.C. 67, 1900 La Plata, Argentina
}

(Received 6 October 2020; accepted 28 October 2020; published 18 November 2020)

\begin{abstract}
We study the description of nucleons and diquarks in the presence of a uniform strong magnetic field within the framework of the two-flavor Nambu-Jona-Lasinio model. Diquarks are constructed through the resummation of quark loop chains using the random phase approximation, while nucleons are treated as bound quark-diquark states described by a relativistic Fadeev equation, using the static approximation for quark exchange interactions. For charged particles, analytical calculations are performed using the Ritus eigenfunction method, which properly takes into account the breakdown of translation invariance that arises from the presence of Schwinger phases. Within this scheme, for definite model parametrizations we obtain numerical predictions for diquark and nucleon masses, which are compared with chiral perturbation theory and lattice QCD results. In addition, numerical estimations for nucleon magnetic moments are obtained.
\end{abstract}

DOI: 10.1103/PhysRevD.102.094020

\section{INTRODUCTION}

In recent years, a significant effort has been devoted to the study of the properties of strongly interacting matter under the influence of strong magnetic fields (see, e.g., [1-3], and references therein). This is mostly motivated by the realization that large magnetic fields might play an important role in the physics of the early Universe [4], in the analysis of high-energy noncentral heavy ion collisions [5], and in the description of physical systems such as magnetars [6]. From the theoretical point of view, addressing this subject requires one to deal with quantum chromodynamics (QCD) in nonperturbative regimes. Therefore, existing analyses are based either in the predictions of effective models or in the results obtained through lattice QCD (LQCD) calculations. Most of these works have been focused on the properties of light mesons. To deal with low-energy QCD, various theoretical approaches have been followed, e.g., NambuJona-Lasinio (NJL)-like models [7-19], quark-meson models [20,21], chiral perturbation theory (ChPT) [22-24], path integral Hamiltonians [25,26], effective chiral confinement Lagrangian approaches [27,28], and QCD sum rules [29].

Published by the American Physical Society under the terms of the Creative Commons Attribution 4.0 International license. Further distribution of this work must maintain attribution to the author(s) and the published article's title, journal citation, and DOI. Funded by SCOAP.
In addition, results for the light meson spectrum in the presence of background magnetic fields have been obtained from LQCD calculations [30-35]. Regarding the study of other hadrons, in the past few years, some works have analyzed the effects of a magnetic field on baryon masses. This problem has been addressed in the context of ChPT [36,37], nonrelativistic quark models [38], extended linear sigma model [39], Walecka model [39,40], soliton models [41], finite energy QCD sum rules [42], and also lattice QCD [43]. It is worth noticing that these theoretical approaches lead to various different results for the behavior of nucleon masses. The main purpose of the present article is to complement these works by studying the effect of an intense external magnetic field on scalar diquark and nucleon properties within the NJL model.

In the framework of the NJL model, mesons and diquarks are usually described as quantum fluctuations in the random phase approximation (RPA) [44-46]; i.e., they are introduced via the summation of an infinite number of quark loops. In the presence of a magnetic field $\vec{B}$, the calculation of these loops requires some care due to the appearance of Schwinger phases [47] associated with quark propagators. For neutral mesons, Schwinger phases cancel out, and, as a consequence, one can take the usual momentum basis to diagonalize the corresponding polarization functions [7-11]. On the other hand, for charged pions and diquarks, the Schwinger phases do not cancel, leading to a breakdown of translational invariance that prevents proceeding as, e.g., in the $\pi^{0}$ case. In this situation, 
some existing calculations $[12,15]$ just neglect Schwinger phases, considering only the translational invariant part of the quark propagators. Recently [16,17], we have introduced a method that allows one to fully take into account the translational-breaking effects introduced by the Schwinger phases in the calculation of charged meson masses within the RPA. This method, based on the Ritus eigenfunction approach [48] to magnetized relativistic systems, allows one to diagonalize the charged pion polarization function in order to obtain the corresponding meson masses. In addition, in Refs. [16,17], we have used a regularization procedure in which only the vacuum contributions to different quantities at zero external magnetic field are regularized. This scheme, that goes under the name of "magnetic-field-independent regularization," has been shown to provide more reliable predictions in comparison with other regularization methods often used in the literature [49]. One of the aims of the present work is to extend the Ritus eigenfunction approach to the case of scalar diquarks. For this purpose, we consider an extended version of the NJL model that includes color pairing interactions.

As mentioned above, another aim of this work is to study the effects of an external magnetic field on nucleon masses. As shown some years ago [50,51], the quark-level NJL Lagrangian can be rewritten in terms of mesonic and baryonic degrees of freedom, using diquarks as effective states in an intermediate step. As a result of the hadronization process, one gets a relativistic Fadeev equation that explicitly takes into account correlations among the three quarks. This equation can be solved numerically in order to determine the nucleon mass [52-55]. In this way, provided that the diquark channel interaction is strong enough, it is seen that one can form a three-quark bound state with a phenomenologically adequate nucleon mass. Using this framework, other nucleon properties have been studied as well [56-58]. In the present work, we will follow this approach, considering the modifications of the aforementioned Fadeev equation induced by the presence of an external magnetic field. As expected, this leads to the existence of two different Fadeev equations, one for the proton and another one for the neutron. Given the complexity of the problem, we consider the static approximation introduced in Ref. [52], which has been shown to lead to an adequate description of nucleon properties in the absence of external fields [54]. Furthermore, for simplicity, we neglect axial vector diquark correlations.

This work is organized as follows. In Sec. II, we introduce the theoretical formalism used to obtain the different quantities we are interested in. In Sec. III, we present and discuss our numerical results. Finally, in
Sec. IV, a summary our work, together with our main conclusions, is given. We also include Appendixes A and B to quote some technical details of our calculations.

\section{THEORETICAL FORMALISM}

\section{A. Bosonized NJL model with diquark interactions in the presence of an external magnetic field}

We start by considering the Euclidean Lagrangian density for the NJL two-flavor model in the presence of an electromagnetic field and color pairing interactions. One has

$$
\begin{aligned}
\mathcal{L}= & \bar{\psi}\left(-i \gamma_{\mu} D_{\mu}+m_{0}\right) \psi-G\left[j^{(S)}(x) j^{(S)}(x)\right. \\
& \left.+j_{a}^{(P)}(x) j_{a}^{(P)}(x)\right]-H\left[j_{A}^{(D)}(x)\right]^{\dagger} j_{A}^{(D)}(x),
\end{aligned}
$$

where $\psi=\left(\psi_{u} \psi_{d}\right)^{T}, G$ and $H$ are coupling constants, and $m_{0}$ is the current quark mass, which is assumed to be equal for $u$ and $d$ quarks. The currents in Eq. (1) are given by

$$
\begin{gathered}
j^{(S)}(x)=\bar{\psi}(x) \psi(x), \\
j_{a}^{(P)}(x)=\bar{\psi}(x) i \gamma_{5} \tau_{a} \psi(x), \\
j_{A}^{(D)}(x)=\bar{\psi}_{c}(x) i \gamma_{5} \tau_{2} \lambda_{A} \psi(x),
\end{gathered}
$$

where we have defined $\psi_{c}=\gamma_{2} \gamma_{4} \bar{\psi}^{T}$, while $\tau_{a}$ and $\lambda_{A}$, with $a=1,2,3$ and $A=2,5,7$, stand for Pauli and Gell-Mann matrices acting on flavor and color spaces, respectively.

The interaction between the fermions and the electromagnetic field $\mathcal{A}_{\mu}$ is driven by the covariant derivative

$$
D_{\mu}=\partial_{\mu}-i \hat{Q} \mathcal{A}_{\mu},
$$

where $\hat{Q}=\operatorname{diag}\left(Q_{u}, Q_{d}\right)$, with $Q_{u}=2 e / 3$ and $Q_{d}=$ $-e / 3$, $e$ being the proton electric charge. We consider the particular case of a homogenous stationary magnetic field $\vec{B}$ orientated along the 3 -axis. Let us choose the Landau gauge, in which $\mathcal{A}_{4}=0, \overrightarrow{\mathcal{A}}=\left(0, B x_{1}, 0\right)$.

To proceed, it is convenient to bosonize the fermionic theory, introducing a scalar field $\sigma(x)$, pseudoscalar fields $\vec{\pi}_{a}(x)$, and diquark fields $\Delta_{A}(x)$, and integrating out the fermion fields. The bosonized Euclidean action can be written as

$$
\begin{aligned}
S_{\mathrm{bos}}= & -\frac{1}{2} \log \operatorname{det} \mathbf{D}+\frac{1}{4 G} \int d^{4} x\left[\sigma(x) \sigma(x)+\pi_{a}(x) \pi_{a}(x)\right] \\
& +\frac{1}{4 H} \int d^{4} x \Delta_{A}(x)^{*} \Delta_{A}(x)
\end{aligned}
$$

where

$$
\mathbf{D}\left(x, x^{\prime}\right)=\delta^{(4)}\left(x-x^{\prime}\right)\left(\begin{array}{cc}
-i \gamma_{\mu} D_{\mu}+m_{0}+\phi(x) & i \gamma_{5} \tau_{2} \lambda_{A} \Delta_{A}(x) \\
i \gamma_{5} \tau_{2} \lambda_{A} \Delta_{A}(x)^{*} & -i \gamma_{\mu} D_{\mu}^{*}+m_{0}+\phi(x)^{T}
\end{array}\right),
$$


with $\phi(x)=\sigma(x)+i \gamma_{5} \tau_{a} \pi_{a}(x)$. As customary, we have used here the Nambu-Gorkov (NG) formalism. In the former equations, and in what follows, matrices in the NG space are denoted in boldface.

We proceed by expanding the bosonized action in powers of the fluctuations $\delta \sigma(x), \delta \pi_{a}(x)$, and $\delta \Delta_{A}(x)$ around the corresponding mean field (MF) values. As usual, we assume that the field $\sigma(x)$ has a nontrivial translational invariant MF value $\bar{\sigma}$, while the vacuum expectation values of pseudoscalar and diquark fields are zero. Then, one has

$$
\mathbf{D}\left(x, x^{\prime}\right)=\overline{\mathbf{D}}\left(x, x^{\prime}\right)+\delta \mathbf{D}\left(x, x^{\prime}\right),
$$

where the MF piece reads

$$
\begin{aligned}
\overline{\mathbf{D}}\left(x, x^{\prime}\right) & =\left(\begin{array}{cc}
\overline{\mathcal{D}}\left(x, x^{\prime}\right) & 0 \\
0 & \overline{\mathcal{D}}_{c}\left(x, x^{\prime}\right)
\end{array}\right) \\
& =\delta^{(4)}\left(x-x^{\prime}\right)\left(\begin{array}{cc}
-i \gamma_{\mu} D_{\mu}+M & 0 \\
0 & -i \gamma_{\mu} D_{\mu}{ }^{*}+M
\end{array}\right) .
\end{aligned}
$$

Here $M$ denotes the quark effective mass, $M=m_{0}+\bar{\sigma}$. The fluctuation piece is given by

$\delta \mathbf{D}\left(x, x^{\prime}\right)=\delta^{(4)}\left(x-x^{\prime}\right)\left(\begin{array}{cc}\delta \phi(x) & i \gamma_{5} \tau_{2} \lambda_{A} \delta \Delta_{A}(x) \\ i \gamma_{5} \tau_{2} \lambda_{A} \delta \Delta_{A}(x)^{*} & \delta \phi(x)^{T}\end{array}\right)$.

The MF operators $\overline{\mathcal{D}}\left(x, x^{\prime}\right)$ and $\overline{\mathcal{D}}_{c}\left(x, x^{\prime}\right)$ are flavor diagonal, and their inverses correspond to quark MF propagators in the presence of a magnetic field. One has

$$
\begin{aligned}
& \overline{\mathcal{D}}^{-1}\left(x, x^{\prime}\right)=\overline{\mathcal{S}}\left(x, x^{\prime}\right)=\operatorname{diag}\left(\overline{\mathcal{S}}^{u}\left(x, x^{\prime}\right), \overline{\mathcal{S}}^{d}\left(x, x^{\prime}\right)\right), \\
& \overline{\mathcal{D}}_{c}^{-1}\left(x, x^{\prime}\right)=\overline{\mathcal{S}}_{c}\left(x, x^{\prime}\right)=\operatorname{diag}\left(\overline{\mathcal{S}}^{-u}\left(x, x^{\prime}\right), \overline{\mathcal{S}}^{-d}\left(x, x^{\prime}\right)\right),
\end{aligned}
$$

where the minus signs in front of the flavor indices $f=u$ or $d$ indicate that the sign of the corresponding quark electric charge in the propagator has to be reversed. As is well known, the explicit form of the quark propagator in the presence of an external constant magnetic field can be written in different ways [2,3]. For convenience, we take the form in which $\overline{\mathcal{S}}^{f}\left(x, x^{\prime}\right)$ is given by a product of a phase factor and a translational invariant function, namely,

$$
\overline{\mathcal{S}}^{f}\left(x, x^{\prime}\right)=e^{i \Phi_{f}\left(x, x^{\prime}\right)} \int_{p_{\perp} p_{\|}} e^{i p\left(x-x^{\prime}\right)} \tilde{S}^{f}\left(p_{\perp}, p_{\|}\right),
$$

where $\Phi_{f}\left(x, x^{\prime}\right)=Q_{f} B\left(x_{1}+x_{1}^{\prime}\right)\left(x_{2}-x_{2}^{\prime}\right) / 2$ is the socalled Schwinger phase. We have introduced here the following shorthand notation for the integrals over twodimensional momentum vectors:

$$
\int_{p q \ldots} \equiv \int \frac{d^{2} p}{(2 \pi)^{2}} \frac{d^{2} q}{(2 \pi)^{2}} \ldots
$$

We find it convenient to express $\tilde{S}^{f}\left(p_{\perp}, p_{\|}\right)$in the Schwinger form $[2,3]$

$$
\begin{aligned}
\tilde{S}^{f}\left(p_{\perp}, p_{\|}\right)= & \int_{0}^{\infty} d \tau e^{-\tau \phi_{f}(\tau, p)}\left\{\left(M-p_{\|} \cdot \gamma_{\|}\right)\right. \\
& \left.\times\left[1+i s_{f} \gamma_{1} \gamma_{2} \tanh \left(\tau B_{f}\right)\right]-\frac{p_{\perp} \cdot \gamma_{\perp}}{\cosh ^{2}\left(\tau B_{f}\right)}\right\},
\end{aligned}
$$

where we have used the following definitions. The "perpendicular" and "parallel" gamma matrices are collected in vectors $\gamma_{\perp}=\left(\gamma_{1}, \gamma_{2}\right)$ and $\gamma_{\|}=\left(\gamma_{3}, \gamma_{4}\right)$, respectively (note that in our convention $\left\{\gamma_{\mu}, \gamma_{\nu}\right\}=-2 \delta_{\mu \nu}$ ). Similarly, $p_{\perp}=\left(p_{1}, p_{2}\right)$ and $p_{\|}=\left(p_{3}, p_{4}\right)$. We have also used the notation $s_{f}=\operatorname{sgn}\left(Q_{f} B\right)$ and $B_{f}=\left|Q_{f} B\right|$. Finally, we have defined

$$
\phi_{f}(\tau, p)=M^{2}+p_{\|}^{2}+\frac{\tanh \left(\tau B_{f}\right)}{\tau B_{f}} p_{\perp}^{2} .
$$

Notice that the integral in Eq. (15) is divergent and has to be properly regularized, as we discuss below.

Replacing the previous relations in the bosonized effective action and expanding in powers of the meson fluctuations around the MF values, one gets

$$
S_{\mathrm{bos}}=S_{\mathrm{bos}}^{\mathrm{MF}}+S_{\mathrm{bos}}^{\mathrm{quad}}+\cdots .
$$

The expression of $S_{\mathrm{bos}}^{\mathrm{MF}}$, together with those of the mesonic contributions to $S_{\text {bos }}^{\text {quad }}$, is given in Eqs. (10)-(12) of Ref. [17]. In that paper, both the procedure followed to obtain the regularized gap equation and the expressions required to calculate various meson properties are discussed in detail. In the present case, $S_{\text {bos }}^{\text {quad }}$ includes an additional contribution that is quadratic in the diquark fields. This contribution will be discussed in the next subsection.

\section{B. Diquark mass and propagator}

The diquark contribution to $S_{\mathrm{bos}}^{\text {quad }}$ is given by

$$
\begin{aligned}
S_{\mathrm{bos}}^{\text {quad,diq }} & =S_{\mathrm{bos}}^{\text {quad }, \Delta}+S_{\mathrm{bos}}^{\mathrm{quad}, \bar{\Delta}} \\
& =\frac{1}{2} \sum_{D=\Delta, \bar{\Delta}} \int d^{4} x d^{4} x^{\prime} \delta D_{A}(x)^{*} \mathcal{G}_{D}^{-1}\left(x, x^{\prime}\right) \delta D_{A}\left(x^{\prime}\right),
\end{aligned}
$$

where 


$$
\mathcal{G}_{D}^{-1}\left(x, x^{\prime}\right)=\frac{1}{4 H} \delta^{(4)}\left(x-x^{\prime}\right)-J_{D}\left(x, x^{\prime}\right)
$$

The polarization functions read

$$
\begin{aligned}
J_{\Delta}\left(x, x^{\prime}\right)= & \operatorname{tr}_{D}\left[\overline{\mathcal{S}}^{u}\left(x, x^{\prime}\right) \gamma_{5} \overline{\mathcal{S}}^{-d}\left(x^{\prime}, x\right) \gamma_{5}\right. \\
& \left.+\overline{\mathcal{S}}^{d}\left(x, x^{\prime}\right) \gamma_{5} \overline{\mathcal{S}}^{-u}\left(x^{\prime}, x\right) \gamma_{5}\right], \\
J_{\bar{\Delta}}\left(x, x^{\prime}\right)= & \operatorname{tr}_{D}\left[\overline{\mathcal{S}}^{-u}\left(x, x^{\prime}\right) \gamma_{5} \overline{\mathcal{S}}^{d}\left(x^{\prime}, x\right) \gamma_{5}\right. \\
& \left.+\overline{\mathcal{S}}^{-d}\left(x, x^{\prime}\right) \gamma_{5} \overline{\mathcal{S}}^{u}\left(x^{\prime}, x\right) \gamma_{5}\right],
\end{aligned}
$$

where the trace is taken over Dirac space. As seen from its quark content, $\Delta(\bar{\Delta})$ corresponds to the diquark with charge $Q_{\Delta}=e / 3\left(Q_{\bar{\Delta}}=-e / 3\right)$. Since $J_{\Delta}\left(x, x^{\prime}\right)=J_{\bar{\Delta}}\left(x^{\prime}, x\right)$, both diquarks have the same mass, and we can proceed by considering only the positively charged diquark $\Delta$.

Let us start by replacing in Eq. (20) the expression for the quark propagators in Eq. (13). We get

$$
\begin{aligned}
J_{\Delta}\left(x, x^{\prime}\right)= & e^{i \Phi_{\Delta}\left(x, x^{\prime}\right)} \int_{p_{\perp} p_{\|} v_{\perp} v_{\|}} e^{i v\left(x-x^{\prime}\right)} \\
& \times \operatorname{tr}_{D}\left[\tilde{S}^{u}\left(p_{\perp}^{+}, p_{\|}^{+}\right) \gamma_{5} \tilde{S}^{-d}\left(p_{\perp}^{-}, p_{\|}^{-}\right) \gamma_{5}\right. \\
& \left.+\tilde{S}^{d}\left(p_{\perp}^{+}, p_{\|}^{+}\right) \gamma_{5} \tilde{S}^{-u}\left(p_{\perp}^{-}, p_{\|}^{-}\right) \gamma_{5}\right]
\end{aligned}
$$

where we have defined $p^{ \pm}=p \pm v / 2$. Here the phase $\Phi_{\Delta}$ is given by

$$
\begin{aligned}
\Phi_{\Delta}\left(x, x^{\prime}\right) & =\Phi_{u}\left(x, x^{\prime}\right)+\Phi_{-d}\left(x^{\prime}, x\right) \\
& =\Phi_{d}\left(x, x^{\prime}\right)+\Phi_{-u}\left(x^{\prime}, x\right) \\
& =\frac{Q_{\Delta} B}{2}\left(x_{1}+x_{1}^{\prime}\right)\left(x_{2}-x_{2}^{\prime}\right) ;
\end{aligned}
$$

i.e., there is no cancellation of Schwinger phases. Consequently, the polarization function is not translational invariant and will not become diagonal when transformed to the momentum basis. In this situation, as done in Ref. [17] for the case of charged pions, it is convenient to expand the diquark field in terms of Ritus eigenfunctions. We have

$$
\delta \Delta_{A}(x)=\bigvee_{\bar{q}} \mathbb{F} \Delta_{\bar{q}}(x) \delta \Delta_{A}(\bar{q})
$$

where we have used the shorthand notation

$$
\bar{q} \equiv\left(\ell, q_{2}, q_{\|}\right), \quad \bigvee_{\bar{q}} \equiv \frac{1}{2 \pi} \sum_{\ell=0}^{\infty} \int \frac{d q_{2}}{2 \pi} \int_{q_{\|}} .
$$

Notice that the expansion includes a sum over discrete Landau levels. The functions $\mathbb{F}_{\bar{q}}^{\Delta}$ are given by
$\mathbb{F}_{\bar{q}}^{\Delta}(x)=N_{\ell} e^{i\left(q_{2} x_{2}+q_{3} x_{3}+q_{4} x_{4}\right)} D_{\ell}\left(\sqrt{2 B_{\Delta}} x_{1}-s_{\Delta} \sqrt{2 B_{\Delta}^{-1}} q_{2}\right)$,

where $D_{\ell}(x)$ are the cylindrical parabolic functions and $N_{\ell}=\left(4 \pi B_{\Delta}\right)^{1 / 4} / \sqrt{\ell}$ !. As in Eq. (15), we use the notation $B_{\Delta}=\left|Q_{\Delta} B\right|$ and $s_{\Delta}=\operatorname{sgn}\left(Q_{\Delta} B\right)$. Replacing now in Eq. (18), we have

$$
S_{\text {bos }}^{\text {quad, } \Delta}=\frac{1}{2} \bigvee_{\bar{q}^{\prime}, \bar{q}} \delta \Delta_{A}(\bar{q})^{*} \mathcal{G}_{\Delta}^{-1}\left(\bar{q}, \bar{q}^{\prime}\right) \delta \Delta_{A}\left(\bar{q}^{\prime}\right),
$$

where

$$
\mathcal{G}_{\Delta}^{-1}\left(\bar{q}, \bar{q}^{\prime}\right)=\frac{1}{4 H} \hat{\delta}_{\bar{q} \bar{q}^{\prime}}-J_{\Delta}\left(\bar{q}, \bar{q}^{\prime}\right)
$$

with

$$
\hat{\delta}_{\bar{q} \bar{q}^{\prime}}=(2 \pi)^{4} \delta_{\ell \ell^{\prime}} \delta\left(q_{2}-q_{2}^{\prime}\right) \delta\left(q_{3}-q_{3}^{\prime}\right) \delta\left(q_{4}-q_{4}^{\prime}\right)
$$

and

$$
\begin{aligned}
J_{\Delta}\left(\bar{q}, \bar{q}^{\prime}\right)= & \int_{p_{\perp} p_{\|} v_{\perp} v_{\|}} \operatorname{tr}_{D}\left[\tilde{S}^{u}\left(p_{\perp}^{+}, p_{\|}^{+}\right) \gamma_{5} \tilde{S}^{-d}\left(p_{\perp}^{-}, p_{\|}^{-}\right) \gamma_{5}\right. \\
& \left.+\tilde{S}^{d}\left(p_{\perp}^{+}, p_{\|}^{+}\right) \gamma_{5} \tilde{S}^{-u}\left(p_{\perp}^{-}, p_{\|}^{-}\right) \gamma_{5}\right] \\
& \times \int d^{4} x d^{4} x^{\prime} e^{i \Phi_{\Delta}\left(x, x^{\prime}\right)} e^{i v\left(x-x^{\prime}\right)} \mathbb{F}_{\bar{q}}^{\Delta}(x)^{*} \mathbb{F}_{\bar{q}^{\prime}}^{\Delta}\left(x^{\prime}\right)
\end{aligned}
$$

The integrals in Eq. (30) can be worked out following basically the same steps as those described in Ref. [17] for the case of charged pions. In this way, after some lengthy calculation, it can be shown that the polarization function turns out to be diagonal in the Ritus eigenfunction basis. One has

$$
J_{\Delta}\left(\bar{q}, \bar{q}^{\prime}\right)=\hat{\delta}_{\bar{q} \bar{q}^{\prime}} J_{\Delta}\left(\ell, \Pi^{2}\right),
$$

where

$$
\begin{aligned}
J_{\Delta}\left(\ell, \Pi^{2}\right)= & \frac{1}{2 \pi^{2}} \int_{0}^{\infty} d z \int_{0}^{1} d y \exp \left[-z M^{2}\right. \\
& \left.-z y(1-y)\left(\Pi^{2}-(2 \ell+1) B_{\Delta}\right)\right] \\
& \times \frac{\alpha_{-}^{\ell}}{\alpha_{+}^{\ell+1}}\left\{\left[M^{2}+\frac{1}{z}\right.\right. \\
& \left.-y(1-y)\left(\Pi^{2}-(2 \ell+1) B_{\Delta}\right)\right]\left(1+t_{u} t_{d}\right) \\
& \left.+\frac{\left(1-t_{u}^{2}\right)\left(1-t_{d}^{2}\right)}{\alpha_{+} \alpha_{-}}\left[\alpha_{-}+\left(\alpha_{-}-\alpha_{+}\right) \ell\right]\right\}
\end{aligned}
$$


with $\Pi^{2}=(2 \ell+1) B_{\Delta}+q_{\|}^{2}$. Here we have introduced the definitions $t_{u}=\tanh \left(B_{u} y z\right), t_{d}=\tanh \left[B_{d}(1-y) z\right]$, and $\alpha_{ \pm}=\left(B_{d} t_{u}+B_{u} t_{d} \pm B_{\Delta} t_{u} t_{d}\right) /\left(B_{u} B_{d}\right)$. As usual, we have introduced the changes of variables $y=\tau /\left(\tau+\tau^{\prime}\right)$ and $z=\tau+\tau^{\prime}, \tau$ and $\tau^{\prime}$ being the integration parameters associated with the quark propagators as in Eq. (15).

As in the case of the mesons [16,17], the polarization function in Eq. (32) turns out to be divergent and can be regularized within the magnetic-field-independent regularization scheme. Because of quantization in the 1-2 plane, this requires some care; viz. the subtraction of the $B=0$ contribution to the polarization function has to be carried out once the latter has been written in terms of the squared canonical momentum $\Pi^{2}$, as in Eq. (32). Thus, the regularized diquark polarization function can be written as

$$
J_{\Delta}^{(\mathrm{reg})}\left(\ell, \Pi^{2}\right)=J_{\Delta, B=0}^{(\mathrm{reg})}\left(\Pi^{2}\right)+J_{\Delta}^{(\mathrm{mag})}\left(\ell, \Pi^{2}\right),
$$

where

$$
\begin{aligned}
J_{\Delta}^{(\mathrm{mag})}\left(\ell, \Pi^{2}\right)= & \frac{1}{2 \pi^{2}} \int_{0}^{\infty} d z \int_{0}^{1} d y \exp \left[-z M^{2}-z y(1-y) \Pi^{2}\right] \\
& \times\left\{\left[M^{2}+\frac{1}{z}-y(1-y)\left[\Pi^{2}-(2 \ell+1) B_{\Delta}\right]\right]\right. \\
& \times\left[\frac{\alpha_{-}^{\ell}}{\alpha_{+}^{\ell+1}}\left(1+t_{u} t_{d}\right) \exp \left[z y(1-y)(2 \ell+1) B_{\Delta}\right]-\frac{1}{z}\right] \\
& +\frac{\alpha_{-}^{\ell-1}}{\alpha_{+}^{\ell+2}}\left(1-t_{u}^{2}\right)\left(1-t_{d}^{2}\right)\left[\alpha_{-}+\left(\alpha_{-}-\alpha_{+}\right) \ell\right] \\
& \left.\times \exp \left[z y(1-y)(2 \ell+1) B_{\Delta}\right]-\frac{1}{z}\left[\frac{1}{z}-y(1-y)(2 \ell+1) B_{\Delta}\right]\right\}
\end{aligned}
$$

The integrand in Eq. (34) is well behaved in the limit $z \rightarrow 0$. Hence, this magnetic-field-dependent contribution is finite. On the other hand, the expression for the subtracted $B=0$ piece has to be regularized. This can be done, as usual, by using a 3D cutoff regularization. We get

$$
J_{\Delta, B=0}^{(\mathrm{reg})}\left(\Pi^{2}\right)=2\left[I_{1}+\Pi^{2} I_{2}\left(\Pi^{2}\right)\right],
$$

where the explicit expressions of $I_{1}$ and $I_{2}$ can be found, e.g., in Ref. [17] [see Eqs. (20) and (28)]. We obtain in this way

$$
\mathcal{G}_{\Delta}^{-1}\left(\bar{q}, \bar{q}^{\prime}\right)=\hat{\delta}_{\bar{q} \bar{q}^{\prime}}\left[\frac{1}{4 H}-J_{\Delta}^{(\mathrm{reg})}\left(\ell, \Pi^{2}\right)\right] .
$$

Since the two-point function is diagonal in this basis, it can be trivially inverted to obtain the diquark propagator. We have

$$
\mathcal{G}_{\Delta}\left(\bar{q}, \bar{q}^{\prime}\right)=\hat{\delta}_{\bar{q} \bar{q}^{\prime}} \mathcal{G}_{\Delta}^{(\mathrm{reg})}\left(\ell, q_{\|}^{2}\right),
$$

where

$$
\mathcal{G}_{\Delta}^{(\mathrm{reg})}\left(\ell, q_{\|}^{2}\right)=\left[\frac{1}{4 H}-J_{\Delta}^{(\mathrm{reg})}\left(\ell, \Pi^{2}\right)\right]^{-1} .
$$

Consequently, in our framework the diquark pole mass in the presence of the magnetic field for each Landau level $\ell$ can be obtained by solving the equation

$$
\frac{1}{4 H}-J_{\Delta}^{(\mathrm{reg})}\left(\ell,-m_{\Delta}^{2}\right)=0 .
$$

It is clear that $m_{\Delta}$ depends on the magnetic field, although not explicitly stated.

As in the case of the charged pions, instead of dealing with $m_{\Delta}$ one can define the $\Delta$ "magnetic-field-dependent mass" as the lowest quantum-mechanically allowed energy of the diquark, $E_{\Delta}$. The latter is given by

$$
E_{\Delta}^{2}=m_{\Delta}^{2}+(2 \ell+1) B_{\Delta}+\left.q_{3}^{2}\right|_{q_{3}=0, \ell=0}=m_{\Delta}^{2}+\frac{|e B|}{3} .
$$

Notice that this "mass" is magnetic field dependent even for a pointlike diquark (in which case one would have a pole mass $m_{\Delta}$ independent of $B$ ). In fact, owing to zero-point motion in the 1-2 plane, even for $\ell=0$ a diquark cannot be at rest in the presence of the magnetic field.

Given the diagonal form of the diquark propagator in Ritus space [see Eq. (37)], we can transform it back to coordinate space. One obtains

$$
\mathcal{G}_{\Delta}\left(x, x^{\prime}\right)=e^{i \Phi_{\Delta}\left(x, x^{\prime}\right)} \int_{q_{\perp} q_{\|}} e^{i q\left(x-x^{\prime}\right)} \tilde{\mathcal{G}}_{\Delta}\left(q_{\perp}, q_{\|}\right),
$$

where 
$\tilde{\mathcal{G}}_{\Delta}\left(q_{\perp}, q_{\|}\right)=2 e^{-q_{\perp}^{2} / B_{\Delta}} \sum_{\ell=0}^{\infty}(-1)^{\ell} \mathcal{G}_{\Delta}^{(\mathrm{reg})}\left(\ell, q_{\|}^{2}\right) L_{\ell}\left(2 q_{\perp}^{2} / B_{\Delta}\right)$,

$L_{\ell}(x)$ being the Laguerre polynomials.

\section{Nucleon masses}

The baryon propagator can be obtained consistently with the bound quark-diquark structure following Ref. [51]. From the infinite sum illustrated by the diagrams in Fig. 1, one arrives at a relation of the form

$$
\begin{aligned}
\mathcal{S}^{B} & \left([x ; y],\left[x^{\prime} ; y^{\prime}\right]\right) \\
= & \mathcal{S}_{0}^{B}\left([x ; y],\left[x^{\prime} ; y^{\prime}\right]\right) \\
& +\int d^{4} t d^{4} z \mathcal{S}_{0}^{B}([x ; y],[t ; z]) \mathcal{H}(z, t) \mathcal{S}_{0}^{B}\left([z ; t],\left[x^{\prime} ; y^{\prime}\right]\right) \\
& +\cdots,
\end{aligned}
$$

where, in our case, the kernel $\mathcal{H}$ is given by

$$
\mathcal{H}(z, t)=i \gamma_{5} \tau_{2} \lambda_{A} \overline{\mathcal{S}}_{c}(z, t) i \gamma_{5} \tau_{2} \lambda_{A^{\prime}}
$$

In Eq. (43), $\mathcal{S}^{B}$ stands for the full baryon propagator, while $\mathcal{S}_{0}^{B}$ describes the unperturbed propagation of a diquark and a quark, namely,

$$
\mathcal{S}_{0}^{B}([x ; y],[t ; z])=\mathcal{G}_{\Delta}(x, t) \overline{\mathcal{S}}(y, z)
$$

Since the nucleon fields are bilocal, we have introduced the notation of pairs $[x ; y]$, where the first and second coordinates correspond to the diquark and the quark, respectively. The resummation of the diagrams in Fig. 1 leads to a relativistic Fadeev equation that can be written in the form

$$
\begin{aligned}
\mathcal{S}_{0}^{B}\left([x ; y],\left[x^{\prime} ; y^{\prime}\right]\right)= & \int d^{4} t d^{4} z\left[\delta^{(4)}(x-z) \delta^{(4)}(y-t)\right. \\
& -L([x ; y],[z ; t])] \mathcal{S}^{B}\left([z ; t],\left[x^{\prime} ; y^{\prime}\right]\right),
\end{aligned}
$$

where

$$
L([x ; y],[z ; t])=\mathcal{S}_{0}^{B}([x ; y],[t ; z]) \mathcal{H}(z, t)
$$

The nucleon masses will be given by the poles of the baryon propagator in the background of the vacuum configuration of the meson fields. These poles correspond to the zeros of the operator in square brackets in Eq. (46). Acting on the baryon field $\psi$, one has

$$
\int d^{4} z d^{4} t L([x ; y],[z ; t]) \psi([z ; t])=\psi([x ; y]) .
$$

It should be noticed that in our calculation only isoscalarscalar diquark interactions have been considered. This implies that the nucleon isospin is directly given by the flavor of the unpaired quark. Projecting on color singlet baryon states, and using the explicit form of the matrices in flavor space, one gets

$$
\begin{aligned}
& 2 \int d^{4} z d^{4} t \mathcal{G}_{\Delta}(x, t) \overline{\mathcal{S}}^{u}(y, z) \gamma_{5} \overline{\mathcal{S}}^{-d}(z, t) \gamma_{5} \psi_{p}([z ; t]) \\
& \quad=\psi_{p}([x ; y]) \\
& 2 \int d^{4} z d^{4} t \mathcal{G}_{\Delta}(x, t) \overline{\mathcal{S}}^{d}(y, z) \gamma_{5} \overline{\mathcal{S}}^{-u}(z, t) \gamma_{5} \psi_{n}([z ; t]) \\
& \quad=\psi_{n}([x ; y]),
\end{aligned}
$$

where $\psi_{p}$ and $\psi_{n}$ stand for the proton and neutron states, respectively.

It should be noticed that in the absence of an external magnetic field both equations coincide. Moreover, since in that case both the quark and diquark fields are translational invariant, one can perform a Fourier transformation into momentum space. The resulting Fadeev equation, discussed, e.g., in Refs. [52,54], turns out to be a nonseparable integral equation. Given its complexity, in Ref. [52], the socalled "static approximation," in which one disregards the momentum dependence of the exchanged quark, was used. Then, in Ref. [54], the full equation was solved numerically, showing that in fact the static approximation can be taken as a good qualitative approach to the exact results. Having this in mind, and taking into account the additional

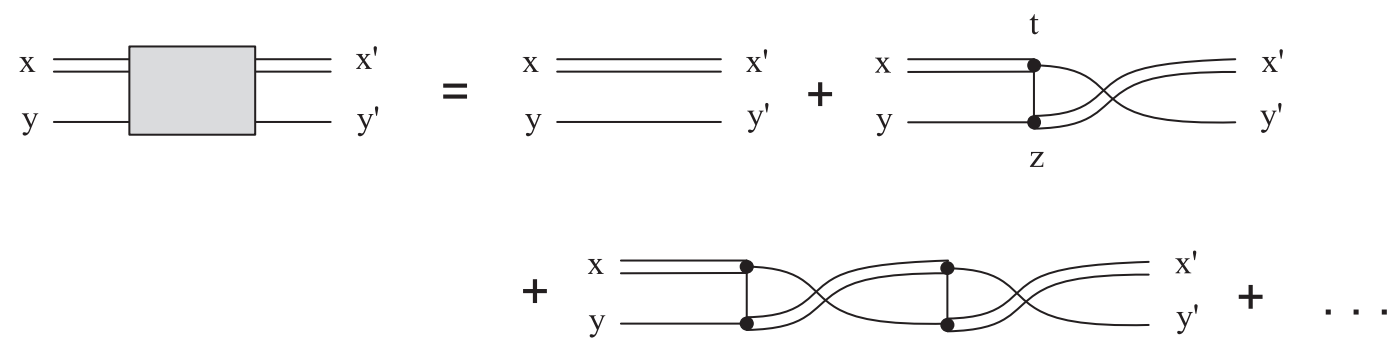

FIG. 1. Diagrams contributing to the full baryon propagator. 
difficulty introduced by the external magnetic field, we find it appropriate to consider the static approximation to get an estimation of the behavior of nucleon masses with the external field. This means to take

$$
\tilde{S}^{-f}\left(p_{\perp}, p_{\|}\right) \rightarrow \frac{1}{M}
$$

Since in this approximation one has $\overline{\mathcal{S}}^{-f}(x, y)=\delta^{(4)}(x-y)$ and $\mathcal{H}(x, z) \propto \delta^{(4)}(x-z)$, Eqs. (50) reduce to

$$
\begin{aligned}
& \frac{2}{M} \int d^{4} z \mathcal{G}_{\Delta}(x, z) \overline{\mathcal{S}}^{u}(x, z) \psi_{p}(z)=\psi_{p}(x), \\
& \frac{2}{M} \int d^{4} z \mathcal{G}_{\Delta}(x, z) \overline{\mathcal{S}}^{d}(x, z) \psi_{n}(z)=\psi_{n}(x) .
\end{aligned}
$$

Notice that within this approximation there is no further need to consider coordinate pairs in the arguments of nucleon fields, which become local.

Inserting Eqs. (13) and (41) into Eqs. (52), we get

$$
\begin{array}{r}
\frac{2}{M} \int_{q_{\perp} q_{\|} r_{\perp} r_{\|}} e^{i(q+r) x} \tilde{\mathcal{G}}_{\Delta}\left(q_{\perp}, q_{\|}\right) \tilde{S}^{u}\left(r_{\perp}, r_{\|}\right) \int d^{4} z e^{i \Phi_{p}(x, z)} e^{-i(q+r) z} \psi_{p}(z)=\psi_{p}(x), \\
\frac{2}{M} \int_{q_{\perp} q_{\|} r_{\perp} r_{\|}} e^{i(q+r) x} \tilde{\mathcal{G}}_{\Delta}\left(q_{\perp}, q_{\|}\right) \tilde{S}^{d}\left(r_{\perp}, r_{\|}\right) \int d^{4} z e^{-i(q+r) z} \psi_{n}(z)=\psi_{n}(x),
\end{array}
$$

where the Schwinger phase appearing in the equation for the proton is given by

$$
\begin{aligned}
\Phi_{p}\left(x, x^{\prime}\right) & =\Phi_{\Delta}\left(x, x^{\prime}\right)+\Phi_{u}\left(x, x^{\prime}\right) \\
& =\frac{Q_{p} B}{2}\left(x_{1}+x_{1}^{\prime}\right)\left(x_{2}-x_{2}^{\prime}\right),
\end{aligned}
$$

with $Q_{p}=e$. As expected, in the equation for the neutron, the Schwinger phase vanishes. In order to change to a momentum basis, it is convenient to introduce the transformations

$$
\begin{aligned}
\psi_{p}(x) & =\bigvee_{\bar{P}} \mathbb{E}_{\bar{P}}^{p}(x) \psi_{p}(\bar{P}), \\
\psi_{n}(x) & =\int \frac{d^{4} P}{(2 \pi)^{4}} e^{i P x_{\psi_{n}}}(P) .
\end{aligned}
$$

Note that, while in the case of the neutron $P$ denotes the usual four-momentum, for the proton field we have used a shorthand notation which resembles the one used for the diquarks, namely,

$$
\bar{P} \equiv\left(k, P_{2}, P_{\|}\right), \quad \bigvee_{\bar{P}} \equiv \frac{1}{2 \pi} \sum_{k=0}^{\infty} \int \frac{d P_{2}}{2 \pi} \int_{P_{\|}} .
$$

The functions $\mathbb{E}_{\bar{P}}^{p}$ are given by

$$
\mathbb{E}_{\bar{P}}^{p}(x)=\sum_{\lambda= \pm} E_{\bar{P}, \lambda}^{p}(x) \Gamma_{\lambda},
$$

where $\Gamma_{+}=\operatorname{diag}(1,0,1,0), \Gamma_{+}=\operatorname{diag}(0,1,0,1)$, and

$$
\begin{aligned}
E_{\bar{P}, \lambda}^{p}(x)= & N_{k_{\lambda}} e^{i\left(P_{2} x_{2}+P_{3} x_{3}+P_{4} x_{4}\right)} \\
& \times D_{k_{\lambda}}\left(\sqrt{2 B_{p}} x_{1}-s_{p} \sqrt{2 B_{p}^{-1}} P_{2}\right) .
\end{aligned}
$$

As in the diquark case, $D_{k_{\lambda}}(x)$ are cylindrical parabolic functions. We have also defined $N_{k_{\lambda}}=\left(4 \pi B_{p}\right)^{1 / 4} / \sqrt{k_{\lambda} !}$, $k_{\lambda}=k-\left(1-\lambda s_{p}\right) / 2, B_{p}=|e B|$, and $s_{p}=\operatorname{sgn}(e B)$.

Equations (53) can be now transformed to momentum space using Eqs. (55). One gets

$$
\begin{aligned}
\sum_{\bar{P}^{\prime}} \mathbb{D}_{\bar{P} \bar{P}^{\prime}}^{(p)} \psi_{p}\left(\bar{P}^{\prime}\right) & =0, \\
\mathbb{D}_{P}^{(n)} \psi_{n}(P) & =0,
\end{aligned}
$$

where

$$
\begin{aligned}
\mathbb{D}_{\bar{P}}^{(p)}= & \hat{\delta}_{\bar{P} \bar{P}^{\prime}} \mathbb{1}-\frac{2}{M} \int_{q_{\perp} q_{\|} r_{\perp} r_{\|}} \sum_{\lambda, \lambda^{\prime}} I_{\bar{P}_{\bar{P}}}^{\lambda, \lambda^{\prime}}(q, r) \tilde{\mathcal{G}}_{\Delta}\left(q_{\perp}, q_{\|}\right) \\
& \times \Gamma_{\lambda} \tilde{S}^{u}\left(r_{\perp}, r_{\|}\right) \Gamma_{\lambda^{\prime}}, \\
\mathbb{D}_{P}^{(n)}= & \mathbb{1}-\frac{2}{M} \int_{q_{\perp} q_{\|}} \tilde{\mathcal{G}}_{\Delta}\left(q_{\perp}, q_{\|}\right) \tilde{S}^{d}\left(P_{\perp}-q_{\perp}, P_{\|}-q_{\|}\right),
\end{aligned}
$$

with

$$
I_{\bar{P}}^{\lambda, \lambda^{\prime}}(q, r)=\int d^{4} x d^{4} z e^{i\left[\Phi_{p}(x, z)+(q+r)(x-z)\right]} E_{\bar{P}, \lambda}^{p}(x)^{*} E_{\bar{P}^{\prime}, \lambda^{\prime}}^{p}(z) .
$$

From Eq. (60), it is not obvious that $\mathbb{D}_{\bar{P} \bar{P}^{\prime}}^{(p)}$ is diagonal in Ritus space. However, after a rather long calculation, it can 
be shown that $\mathbb{D}_{\bar{P} \bar{P}^{\prime}}^{(p)}$ is indeed proportional to $\hat{\delta}_{\bar{P} \bar{P}^{\prime}}$. The main steps of the calculation are given in Appendix A. Using the form of the quark propagator given in Eq. (15), one finally obtains

$$
\begin{aligned}
& \mathbb{D}_{\bar{P} \bar{P}^{\prime}}^{(p)}=\hat{\delta}_{\bar{P} \bar{P}^{\prime}} \sum_{\lambda= \pm}\left[X_{\lambda}^{(p)}+Y_{\lambda}^{(p)} P_{\|} \cdot \gamma_{\|}+Z_{\lambda}^{(p)} \gamma_{2}\right] \Gamma_{\lambda}, \\
& \mathbb{D}_{P}^{(n)}=\sum_{\lambda= \pm}\left[X_{\lambda}^{(n)}+Y_{\lambda}^{(n)} P_{\|} \cdot \gamma_{\|}+Z^{(n)} P_{\perp} \cdot \gamma_{\perp}\right] \Gamma_{\lambda},
\end{aligned}
$$

where

$$
\begin{aligned}
X_{\lambda}^{(p)}= & 1-\frac{8 \pi}{B_{p}}(-1)^{k_{\lambda}} \int_{q_{\perp} q_{\|} r_{\perp}} e^{-\left(q_{\perp}+r_{\perp}\right)^{2} / B_{p}} \tilde{\mathcal{G}}_{\Delta}\left(q_{\perp}, q_{\|}\right) \\
& \times T_{\lambda}^{u}\left(r_{\perp}, P_{\|}-q_{\|}\right) L_{k_{\lambda}}\left(\frac{2\left(r_{\perp}+q_{\perp}\right)^{2}}{B_{p}}\right), \\
Y_{\lambda}^{(p)}= & \frac{8 \pi}{M B_{p}}(-1)^{k_{\lambda}} \int_{q_{\perp} q_{\|} r_{\perp}} e^{-\left(q_{\perp}+r_{\perp}\right)^{2} / B_{p}} \tilde{\mathcal{G}}_{\Delta}\left(q_{\perp}, q_{\|}\right) \\
& \times\left(1-\frac{q_{\|} \cdot P_{\|}}{P_{\|}^{2}}\right) T_{\lambda}^{u}\left(r_{\perp}, P_{\|}-q_{\|}\right) L_{k_{\lambda}}\left(\frac{2\left(r_{\perp}+q_{\perp}\right)^{2}}{B_{p}}\right), \\
Z_{\lambda}^{(p)}= & \frac{8 \pi s_{p}}{M B_{p}} \sqrt{\frac{2}{k B_{p}}(-1)^{k}} \int_{q_{\perp} q_{\|} r_{\perp}} e^{-\left(q_{\perp}+r_{\perp}\right)^{2} / B_{p}} \tilde{\mathcal{G}}_{\Delta}\left(q_{\perp}, q_{\|}\right) \\
& \times r_{\perp}\left[\left(r_{1}+q_{1}\right)-i \lambda\left(r_{2}+q_{2}\right)\right] V^{u}\left(r_{\perp}, P_{\|}-q_{\|}\right) \\
& \times L_{k-1}^{1}\left(\frac{2\left(r_{\perp}+q_{\perp}\right)^{2}}{B_{p}}\right),
\end{aligned}
$$

and

$$
X_{\lambda}^{(n)}=1-2 \int_{q_{\perp} q_{\|}} \tilde{\mathcal{G}}_{\Delta}\left(q_{\perp}, q_{\|}\right) T_{\lambda}^{d}\left(P_{\perp}-q_{\perp}, P_{\|}-q_{\|}\right),
$$

$$
\begin{aligned}
Y_{\lambda}^{(n)}= & \frac{2}{M} \int_{q_{\perp} q_{\|}} \tilde{\mathcal{G}}_{\Delta}\left(q_{\perp}, q_{\|}\right) T_{\lambda}^{d}\left(P_{\perp}-q_{\perp}, P_{\|}-q_{\|}\right) \\
& \times\left(1-\frac{q_{\|} \cdot P_{\|}}{P_{\|}^{2}}\right),
\end{aligned}
$$$$
Z_{\lambda}^{(n)}=\frac{2}{M} \int_{q_{\perp} q_{\|}} \tilde{\mathcal{G}}_{\Delta}\left(q_{\perp}, q_{\|}\right) V^{d}\left(P_{\perp}-q_{\perp}, P_{\|}-q_{\|}\right)
$$$$
\times\left(1-\frac{q_{\perp} \cdot P_{\perp}}{P_{\perp}^{2}}\right)
$$

with

$$
\begin{aligned}
& T_{\lambda}^{f}\left(r_{\perp}, r_{\|}\right)=\int_{0}^{\infty} d \tau e^{-\tau \phi_{f}(\tau, r)}\left[1+\lambda s_{f} \tanh \left(\tau B_{f}\right)\right], \\
& V^{f}\left(r_{\perp}, r_{\|}\right)=\int_{0}^{\infty} d \tau e^{-\tau \phi_{f}(\tau, r)} \operatorname{sech}^{2}\left(\tau B_{f}\right) .
\end{aligned}
$$

In what follows, we will concentrate on the determination of the proton and neutron lowest possible energies. Since these quantities are usually interpreted as the nucleon masses, we denote them as $\mathcal{M}_{N}$, with $N=p, n$. For the neutron we just take, as usual, $\vec{P}_{\perp}=0, P_{3}=0$, and $P_{4}^{2}=-\mathcal{M}_{n}^{2}$. In the case of the proton, as done for the diquarks, we consider the squared canonical momentum $\Pi^{2}=2 k B_{p}+P_{\|}^{2}$. The lowest-energy state corresponds to the lowest Landau level (LLL), $k=0$. Then, taking $P_{3}=0$, one has $P_{4}^{2}=-\mathcal{M}_{p}^{2}$, as for the neutron case. Since the determinants of the Dirac operators in Eqs. (63) have to vanish at the pole masses, the corresponding eigenvalue equations read

$$
\begin{aligned}
& \hat{X}_{s_{p}}^{(p) 2}-\mathcal{M}_{p}^{2} \hat{Y}_{s_{p}}^{(p) 2}=0, \\
& \hat{X}_{\lambda}^{(n) 2}-\mathcal{M}_{n}^{2} \hat{Y}_{\lambda}^{(n) 2}=0,
\end{aligned}
$$

where we have denoted by $\hat{X}_{ \pm}^{(N)}$ and $\hat{Y}_{ \pm}^{(N)}$ the coefficients in Eqs. (63) evaluated at $k=0, P_{3}=0$, and $\vec{P}_{\perp}=0$. Note that for the lowest-energy states there is no contribution from the terms with $Z_{\lambda}^{(p)}$ and $Z_{\lambda}^{(n)}$. In addition, in the case of the proton only the projection $\lambda=s_{p}$ is nonvanishing for $k=0$. For the neutron, both projections are, in principle, allowed, and one should take the value of $\lambda$ that leads to the lowest value of the mass.

To obtain the explicit form of the coefficients $\hat{X}_{\lambda}^{(N)}$ and $\hat{Y}_{\lambda}^{(N)}$ needed to evaluate- and solve-Eqs. (71) and (72), one has to replace the diquark propagator, Eq. (42), in Eqs. (64), (65), (67), and (68). For convenience, we consider first the form of the coefficients in the absence of the external magnetic field (in this case, both proton and neutron are taken at rest). They are given by (see Appendix B)

$$
\begin{aligned}
\hat{X}= & 1-\frac{1}{4 \pi^{2} m_{N}} \int_{1}^{\infty} \frac{d \tau}{\tau} \int_{0}^{\infty} d q q^{2} \mathcal{G}_{\Delta, B=0}^{(\mathrm{reg})}\left(q^{2}\right) \\
& \times e^{-\tau\left(M^{2}+q^{2}-m_{N}^{2}\right) / \Lambda_{B}^{2}} J_{1}\left(\frac{2 \tau q m_{N}}{\Lambda_{B}^{2}}\right), \\
\hat{Y}= & \frac{1}{4 \pi^{2} m_{N} M} \int_{1}^{\infty} \frac{d \tau}{\tau} \int_{0}^{\infty} d q q^{2} \mathcal{G}_{\Delta, B=0}^{(\mathrm{reg})}\left(q^{2}\right) e^{-\tau\left(M^{2}+q^{2}-m_{N}^{2}\right) / \Lambda_{B}^{2}} \\
\times & {\left[J_{1}\left(\frac{2 \tau q m_{N}}{\Lambda_{B}^{2}}\right)-\frac{q}{m_{N}} J_{2}\left(\frac{2 \tau q m_{N}}{\Lambda_{B}^{2}}\right)\right] . }
\end{aligned}
$$

Here, and below, $m_{N}$ denotes the nucleon mass at $B=0$, and $J_{k}(x)$ are Bessel functions. The $B=0$ diquark propagator [see Eq. (38)] is given by 


$$
\mathcal{G}_{\Delta, B=0}^{(\mathrm{reg})}\left(q^{2}\right)=\left[\frac{1}{4 H}-J_{\Delta, B=0}^{(\mathrm{reg})}\left(q^{2}\right)\right]^{-1} .
$$

Notice that Eqs. (73) and (74) include a cutoff parameter $\Lambda_{B}$, which has been introduced in order to regularize the otherwise divergent quark-diquark loop within the proper time regularization scheme.

For nonzero magnetic field $B$, in the case of the proton we have

$$
\begin{gathered}
\hat{X}_{s_{p}}^{(p)}=1-\frac{B_{u} B_{\Delta}}{2 \pi^{2} \Lambda_{B}^{2}} \int_{1}^{\infty} d \tau \frac{1+t_{u}}{B_{u}+\left(B_{p}+B_{\Delta}\right) t_{u}} \sum_{\ell=0}^{\infty}\left[\frac{B_{u}+\left(B_{p}-B_{\Delta}\right) t_{u}}{B_{u}+\left(B_{p}+B_{\Delta}\right) t_{u}}\right]^{\ell} \\
\times \int_{0}^{\infty} d q_{\|} q_{\|} \mathcal{G}_{\Delta}^{(\mathrm{reg})}\left(\ell, q_{\|}^{2}\right) e^{-\tau\left(M^{2}+q_{\|}^{2}-\mathcal{M}_{p}^{2}\right) / \Lambda_{B}^{2}} J_{0}\left(\frac{2 \tau q_{\|} \mathcal{M}_{p}}{\Lambda_{B}^{2}}\right), \\
\hat{Y}_{s_{p}}^{(p)}=\frac{B_{u} B_{\Delta}}{2 \pi^{2} M \Lambda_{B}^{2}} \int_{1}^{\infty} d \tau \frac{1+t_{u}}{B_{u}+\left(B_{p}+B_{\Delta}\right) t_{u}} \sum_{\ell=0}^{\infty}\left[\frac{B_{u}+\left(B_{p}-B_{\Delta}\right) t_{u}}{B_{u}+\left(B_{p}+B_{\Delta}\right) t_{u}}\right]^{\ell} \\
\times \int_{0}^{\infty} d q_{\|} q_{\|} \mathcal{G}_{\Delta}^{(\mathrm{reg})}\left(\ell, q_{\|}^{2}\right) e^{-\tau\left(M^{2}+q_{\|}^{2}-\mathcal{M}_{p}^{2}\right) / \Lambda_{B}^{2}}\left[J_{0}\left(\frac{2 \tau q_{\|} m_{p}}{\Lambda_{B}^{2}}\right)-\frac{q_{\|}}{\mathcal{M}_{p}} J_{1}\left(\frac{2 \tau q_{\|} \mathcal{M}_{p}}{\Lambda_{B}^{2}}\right)\right],
\end{gathered}
$$

while for the neutron we get

$$
\begin{gathered}
\hat{X}_{\lambda}^{(n)}=1-\frac{B_{d} B_{\Delta}}{2 \pi^{2} \Lambda_{B}^{2}} \int_{1}^{\infty} d \tau \frac{1+\lambda s_{d} t_{d}}{B_{d}+B_{\Delta} t_{d}} \sum_{\ell=0}^{\infty}\left[\frac{B_{d}-B_{\Delta} t_{d}}{B_{d}+B_{\Delta} t_{d}}\right]^{\ell} \\
\times \int_{0}^{\infty} d q_{\|} q_{\|} \mathcal{G}_{\Delta}^{(\mathrm{reg})}\left(\ell, q_{\|}^{2}\right) e^{-\tau\left(M^{2}+q_{\|}^{2}-\mathcal{M}_{n}^{2}\right) / \Lambda_{B}^{2}} J_{0}\left(\frac{2 \tau q_{\|} \mathcal{M}_{n}}{\Lambda_{B}^{2}}\right), \\
\hat{Y}_{\lambda}^{(n)}=\frac{B_{d} B_{\Delta}}{2 \pi^{2} M \Lambda_{B}^{2}} \int_{1}^{\infty} d \tau \frac{1+\lambda s_{d} t_{d}}{B_{d} B_{\Delta} t_{d}} \sum_{\ell=0}^{\infty}\left[\frac{B_{d}-B_{\Delta} t_{d}}{B_{d}+B_{\Delta} t_{d}}\right]^{\ell} \\
\times \int_{0}^{\infty} d q_{\|} q_{\|} \mathcal{G}_{\Delta}^{(\mathrm{reg})}\left(\ell, q_{\|}^{2}\right) e^{-\tau\left(M^{2}+q_{\|}^{2}-\mathcal{M}_{n}^{2}\right) / \Lambda_{B}^{2}}\left[J_{0}\left(\frac{2 \tau q_{\|} \mathcal{M}_{n}}{\Lambda_{B}^{2}}\right)-\frac{q_{\|}}{\mathcal{M}_{n}} J_{1}\left(\frac{2 \tau q_{\|} \mathcal{M}_{n}}{\Lambda_{B}^{2}}\right)\right] .
\end{gathered}
$$

In these equations, we have used the definition $t_{f}=\tanh \left(\tau B_{f} / \Lambda_{B}^{2}\right)$.

\section{Nucleon magnetic moments}

We finish this section by noting that, given the above expressions for $\hat{X}_{\lambda}^{(N)}$ and $\hat{Y}_{\lambda}^{(N)}$, they can be expanded around $B=0$ in order to study how nucleon masses get modified to lowest order in the magnetic field. Let us define the corresponding slopes $\alpha_{N}$ by

$$
\mathcal{M}_{N}=m_{N}+\alpha_{N}|B|+\mathcal{O}\left(B^{2}\right) .
$$

After a rather long calculation, sketched in Appendix B, we obtain

$$
\begin{aligned}
& \alpha_{p}=\frac{-Q_{u}\left[\left(M+m_{N}\right) \mathcal{I}_{1}-m_{N} \mathcal{I}_{2}\right]+Q_{p} \hat{W}}{M \hat{Y}+2 m_{N} \hat{W}}, \\
& \alpha_{n}=\frac{Q_{d}\left[\left(M+m_{N}\right) \mathcal{I}_{1}-m_{N} \mathcal{I}_{2}\right]}{M \hat{Y}+2 m_{N} \hat{W}}
\end{aligned}
$$

where we have defined

$$
\hat{W}=\left(M+m_{N}\right) \mathcal{I}_{1}-\left(2 m_{N}+M\right) \mathcal{I}_{2}+m_{N} \mathcal{I}_{3},
$$

and the integrals $\mathcal{I}_{k}$ are given by

$$
\begin{aligned}
\mathcal{I}_{k}= & \frac{1}{4 \pi^{2} \Lambda_{B}^{2} m_{N}^{k}} \int_{1}^{\infty} d \tau \int_{0}^{\infty} d q q^{k+1} \mathcal{G}_{\Delta, B=0}\left(q^{2}\right) \\
& \times e^{-\tau\left(M^{2}+q^{2}-m_{N}^{2}\right) / \Lambda_{B}^{2}} J_{k}\left(\frac{2 \tau q m_{N}}{\Lambda_{B}^{2}}\right) .
\end{aligned}
$$

To find the relation between $\alpha_{N}$ and the nucleon magnetic moments, we proceed as follows. First, we take into account that to leading order in the magnetic field the change in the nucleon energy is given by [36,59]

$$
\Delta E_{N}=\frac{\left|Q_{N} B\right|}{2 m_{N}}-\vec{\mu}_{N} \cdot \vec{B}+\mathcal{O}\left(B^{2}\right)
$$

The first term corresponds to orbital motion. While it vanishes for the neutron, for the proton it provides a contribution due to zero-point motion in the plane perpendicular to the magnetic field. The second term 
represents, for both $p$ and $n$, the spin contribution leading to the Zeeman effect. Thus, we have

$$
\begin{aligned}
\Delta E_{p} & =\left(1-\mu_{p}\right) \frac{e|B|}{2 m_{N}}+\mathcal{O}\left(B^{2}\right), \\
\Delta E_{n} & =-\lambda \mu_{n} \frac{e B}{2 m_{N}}+\mathcal{O}\left(B^{2}\right),
\end{aligned}
$$

where, as usual, the nucleon magnetic moments are expressed in units of the nuclear magneton $\mu_{N}=e /\left(2 m_{N}\right)$. Note that for the proton we have taken into account the fact that for the lowest-energy state one has $\lambda=s_{p}$. In this way, identifying the corresponding slopes at $B=0$, the nucleon magnetic moments are given by

$$
\begin{aligned}
& \mu_{p}=1-\frac{2 m_{N}}{e} \alpha_{p}, \\
& \mu_{n}=-\lambda \operatorname{sgn}(B) \frac{2 m_{N}}{e} \alpha_{n} .
\end{aligned}
$$

\section{NUMERICAL RESULTS}

To obtain numerical results for diquark and baryon properties, one has to fix the model parametrization. Here, as done in Ref. [16], we take the parameter set $m_{0}=5.66 \mathrm{MeV}, \Lambda=613.4 \mathrm{MeV}$, and $G \Lambda^{2}=2.250$, which (for a vanishing external field) corresponds to a constituent quark mass $M=350 \mathrm{MeV}$ and a quark-antiquark condensate $\langle\bar{f} f\rangle=(-243.3 \mathrm{MeV})^{3}$. This parametrization properly reproduces the empirical values of the pion mass and decay constant in vacuum, $m_{\pi}=138 \mathrm{MeV}$ and $f_{\pi}=92.4 \mathrm{MeV}$. It also provides a very good agreement with the results from lattice QCD quoted in Ref. [30] for the normalized average $\bar{f} f$ condensate $\Delta \bar{\Sigma}(B)$ up to $|e B| \simeq 1 \mathrm{GeV}^{2}$ [16]. The effective Lagrangian in Eq. (1) also includes the scalar quark-quark coupling constant $H$. Typical effective approaches for the strong interaction, such as the one-gluon exchange or the instanton liquid model, lead to $H / G=0.75$ [60]. However, this value is subject to somewhat large uncertainties from the phenomenological point of view. In fact, larger values for this ratio seem to be favored from the determination of baryon properties within the Fadeev approach [52-55]. Here we choose to take $H / G$ within the range $0.75 \leq H / G \leq 1.2$, typically considered in the literature. The corresponding values of the diquark mass and binding energies are shown in Fig. 2. We observe that, for $H / G \simeq 0.75$ the scalar diquark is barely bound by $5 \mathrm{MeV}$, while for $H / G=1.2$ one gets binding energies of about $200 \mathrm{MeV}$.

Let us consider the magnetic field dependence of the diquark mass. In the upper panel in Fig. 3, we show the values of $m_{\Delta}$ for the LLL [defined by Eq. (39), with $\ell=0$ ] relative to the values obtained for vanishing magnetic field $m_{\Delta, 0}$ as functions of $B_{e}=|e B|$. The curves correspond to some selected values of the ratio $H / G$ within the range
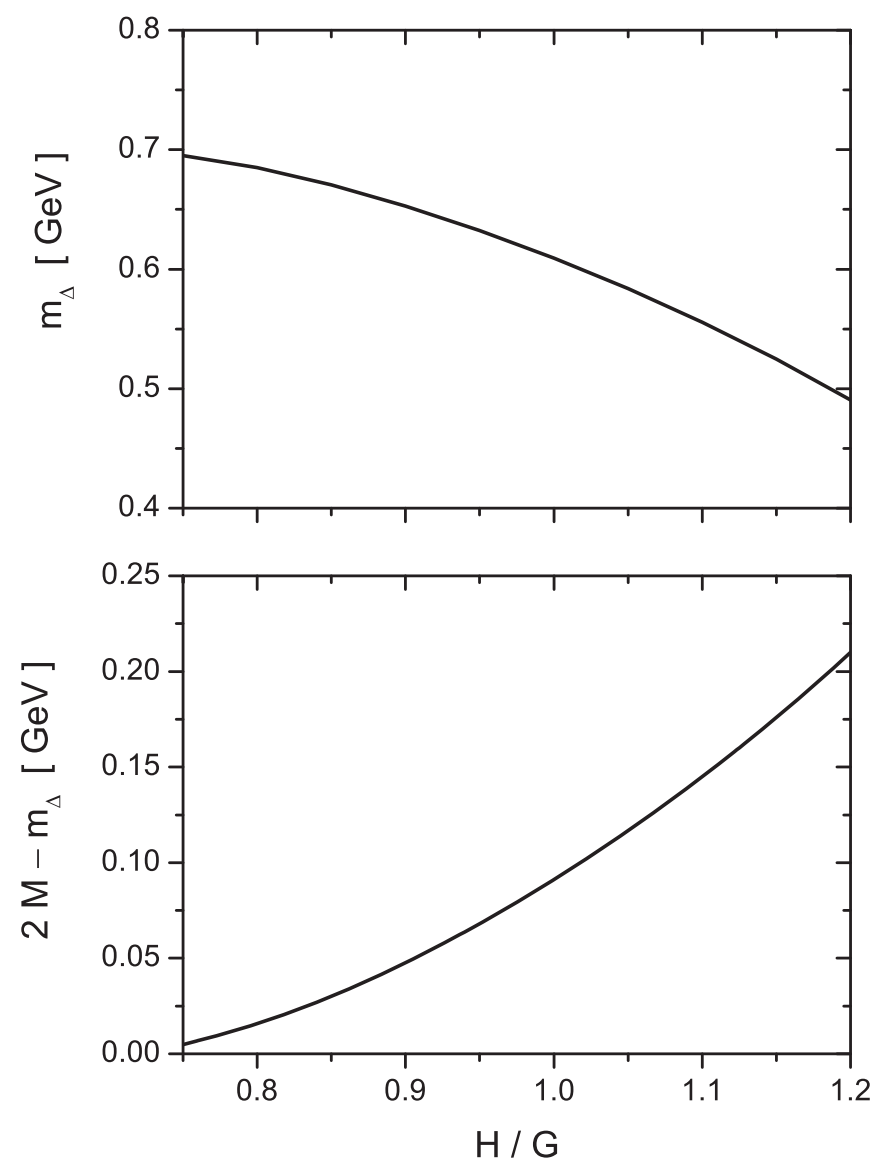

FIG. 2. $\Delta$ mass (top) and binding energy (bottom) at $B=0$ as functions of $H / G$.

mentioned above. We get $m_{\Delta, 0}=0.685,0.653,0.609$, and $0.555 \mathrm{GeV}$ for $H / G=0.8,0.9,1.0$, and 1.1 , respectively. It is seen that for all considered values of $H / G$ the curves start with a decrease of $m_{\Delta}$ as $B_{e}$ increases, reaching a minimum at about $B_{e} \sim 0.2 \mathrm{GeV}^{2}$. Beyond this minimum, the diquark pole mass steadily increases with the magnetic field, reaching a ratio $m_{\Delta} / m_{\Delta, 0}=1$ somewhere in the range $B_{e} \sim 0.4-0.6 \mathrm{GeV}^{2}$, depending on the precise value of $H / G$. In the lower panel in Fig. 3, we show the behavior of the squared magnetic-field-dependent diquark mass $E_{\Delta}^{2}$ [defined by Eq. (40)], minus the corresponding value at $B=0, m_{\Delta, 0}^{2}$. We recall that in the case of a pointlike diquark the mass $m_{\Delta}$ does not depend on the magnetic field, and the difference $E_{\Delta}^{2}-m_{\Delta, 0}^{2}$ is simply given by $B_{e} / 3$. Such a case is indicated by the straight dotted black line. It can be observed that, as a consequence of the initial decrease of the pole mass, for small values of $B_{e}$ the difference $E_{\Delta}^{2}-m_{\Delta, 0}^{2}$ lies below that straight line. At the point in which $m_{\Delta}=m_{\Delta, 0}$, the situation reverses, and for larger values of $B_{e}$ the value of $E_{\Delta}$ becomes larger than in the case of a pointlike diquark. We notice that a similar behavior was found in the analysis of Ref. [15], where Schwinger phases were not taken into account. However, in 

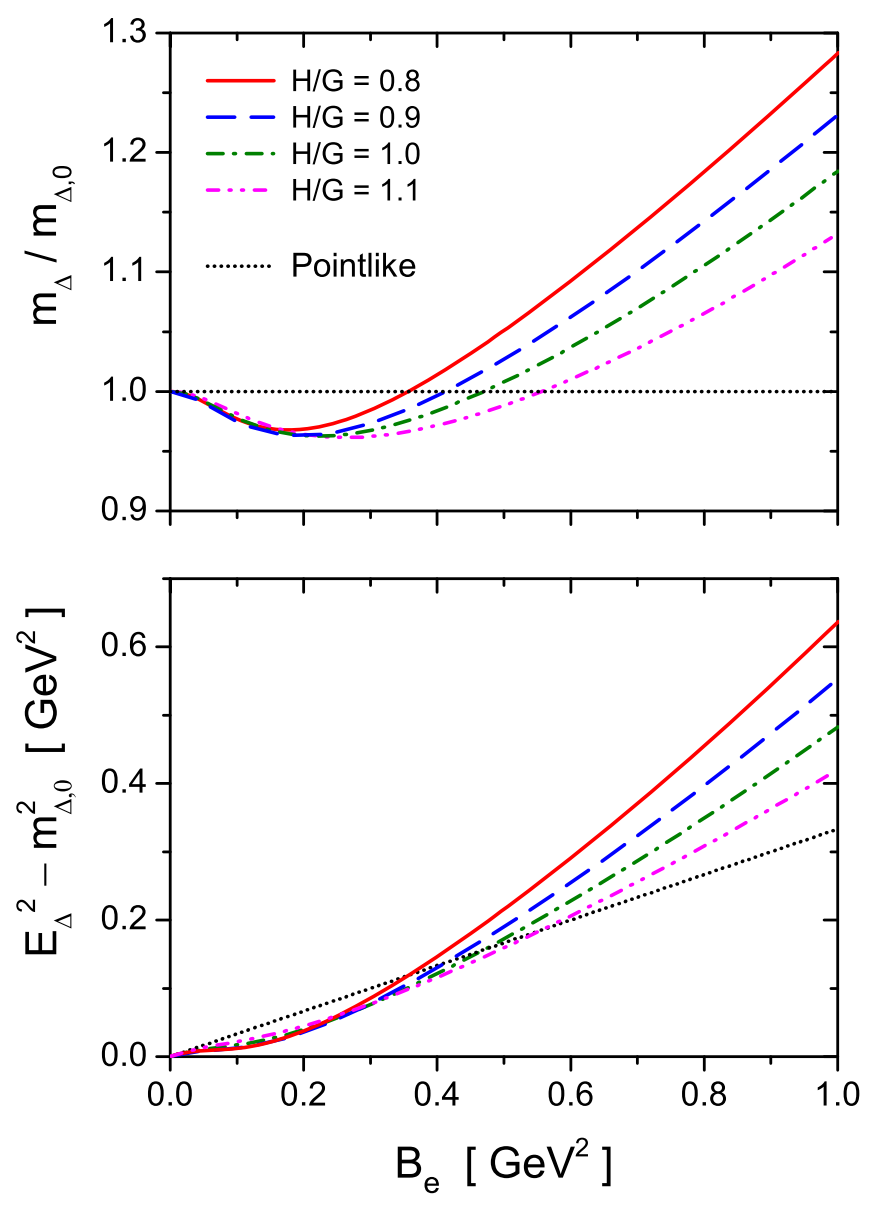

FIG. 3. Relative values of diquark mass and energy as functions of $|e B|$ for some representative values of $H / G$. The results for the case of a pointlike diquark are indicated by the dotted lines.

that work, the crossing was found to occur at a larger value of $B_{e}$, of about $0.9 \mathrm{GeV}^{2}$ for $H / G=0.75$. It is interesting to note that as $H / G$ increases the behavior of $E_{\Delta}^{2}-m_{\Delta, 0}^{2}$ gets closer to the pointlike case. This might be understood by realizing that a larger value of $H / G$ implies a more deeply bound diquark and, consequently, a more localized one.

We turn next to the analysis of nucleon masses. As mentioned in Sec. III.C, the calculation of these quantities requires the introduction of an additional cutoff parameter $\Lambda_{B}$ to regularize the otherwise divergent quark-diquark loop in the proper time regularization scheme. For a given value of $H / G$, we adjust this parameter, demanding the $B=0$ eigenvalue equation $|\hat{X}|=m_{N}|\hat{Y}|$ [see Eqs. (73) and (74)] to be satisfied for the physical value $m_{N}=0.938 \mathrm{GeV}$. In this way, we obtain $\Lambda_{B}=1.618,1.380$, and $1.104 \mathrm{GeV}$ for $H / G=0.8,0.9$, and 1.0 , respectively. For larger values of $H / G$, no value of $\Lambda_{B}$ is found to be compatible with the physical nucleon mass at zero magnetic field in this model. Having determined all input parameters, one can solve the eigenvalue equations (71) and (72) to obtain proton and neutron masses for a nonvanishing external magnetic field.
Before reporting the corresponding results, we find it convenient to make a few comments concerning the numerical details of the calculation. First, we note that to evaluate the coefficients $\hat{X}_{ \pm}^{(N)}$ and $\hat{Y}_{ \pm}^{(N)}$ in Eqs. (76)-(79) one has to perform a sum over Landau levels (LLs). In that sum, we have taken into account as many LLs as needed in order to obtain a stable result for the calculated mass. For low values of $B_{e}$, this implies the inclusion of a quite large number of LLs. For example, at $B_{e}=0.04 \mathrm{GeV}^{2}$, for $H / G=1$ about 300 LLs are needed in order to obtain an accuracy of about $1 \mathrm{MeV}$ in the nucleon mass. For $H / G=0.8$ the required number of LLs is found to be even larger, of the order of 600. As expected, for larger values of the magnetic field the needed number of LLs gets significantly reduced. Still, it is found that for $B_{e}$ as large as $0.8 \mathrm{GeV}^{2}$ about ten LLs are needed to obtain the above mentioned accuracy in the mass determination. Another issue that requires some care is the numerical evaluation of the integrals in Eqs. (76)-(79), due to the highly oscillatory behavior of the Bessel functions for large values of their arguments.

Our results for the behavior of nucleon masses as functions of the external magnetic field are given in Fig. 4. In the upper (lower) panel, we quote the curves for the proton (neutron) mass, considering $H / G=0.8,0.9$, and 1.0. In all cases, it is seen that the masses initially decrease when the magnetic field is increased, reaching a minimum for a value of $B_{e}$ that depends on the parameter $H / G$. Beyond that point, the masses show a steady growth. For both proton and neutron masses, the decrease becomes less pronounced (and the minimum occurs at smaller $B_{e}$ ) the larger the value of $H / G$ is. It is also seen that the dependence on $H / G$ is weaker in the case of the neutron. Let us recall that for a proton in the LLL only the spin projection $\lambda=s_{p}=\operatorname{sgn}\left(Q_{p} B\right)$ is allowed, while both values of $\lambda$ are allowed for the neutron. In Fig. 4, we have plotted the values corresponding to the lower solution of Eq. (72), defined as the neutron mass. In our model, for $B>0(B<0)$ it is found that this lower state corresponds to $\lambda=-1(\lambda=1)$. For the higher state, not shown in the figure, it is seen that the value of $\mathcal{M}_{n}$ obtained as a solution of Eq. (72) initially increases with $B_{e}$. This solution is found to exist only for $B_{e} \lesssim 0.1-0.2 \mathrm{GeV}^{2}$ (the state becomes unbound for larger values of the external field).

As stated, close to $B=0$ both proton and neutron masses are shown to decrease for an increasing external field; i.e., the slopes $\alpha_{p}$ and $\alpha_{n}$ obtained from Eq. (81) are found to be negative. Taking into account that for the lowest neutron state one has $\lambda \operatorname{sgn}(B)=-1$, from Eqs. (86) one gets $\mu_{p}>0$ and $\mu_{n}<0$, as expected from phenomenology. In addition, the fact that the curves show negative slopes at $B=0$ is consistent with the results from ChPT quoted in Ref. [37]. The latter, which are expected to hold for low values of the external field, are shown by dotted lines in Fig. 4. Notice, however, that the slopes obtained within 

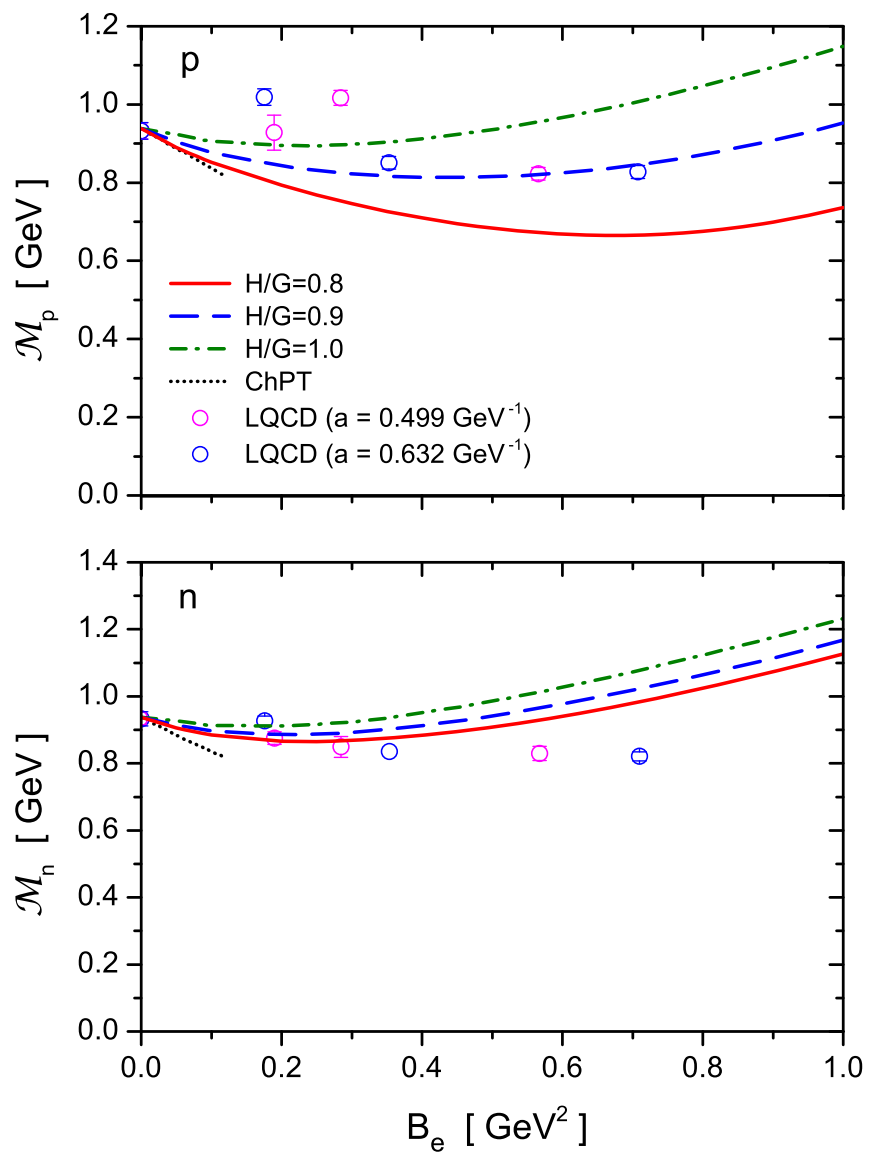

FIG. 4. Proton and neutron masses as functions of $|e B|$ for various values of $H / G$. Open dots and dotted lines correspond to lattice QCD results given in Ref. [43] and ChPT results given in Ref. [37], respectively.

ChPT are, in general, steeper that those found from our results. The lower slopes in our model imply, in turn, relatively low results for the absolute values of proton and neutron magnetic moments. From the numerical evaluation of Eqs. (81) and (86), we find the magnetic moments quoted in Table I, to be compared with the empirical values $\mu_{p}=2.79$ and $\mu_{n}=-1.91$. In this regard, it should be stressed that in our work we have neglected for simplicity the axial vector diquark correlations. The latter can be important to get an enhancement in $\left|\mu_{p}\right|$ and $\left|\mu_{n}\right|$, as shown in Ref. [58]. Finally, let us compare our results with those obtained from LQCD calculations. In Fig. 4, we have indicated with open dots the results from LQCD quoted in

TABLE I. Predicted values of nucleon magnetic moments for different values of $H / G$.

\begin{tabular}{lcc}
\hline \hline$H / G$ & $\mu_{p}$ & $\mu_{n}$ \\
\hline 0.8 & 2.63 & -1.19 \\
0.9 & 2.30 & -1.05 \\
1.0 & 1.99 & -0.94 \\
\hline \hline
\end{tabular}

Ref. [43], corresponding to two different values of the lattice spacing $a$. We observe some qualitative agreement with our results, although LQCD values tend to show a lower dependence on the external field. In the case of the proton, a few lattice points seem to show a mass enhancement for $B_{e} \simeq 0.2-0.3 \mathrm{GeV}^{2}$. Presumably, this could be due to the fact that, as mentioned by the authors of Ref. [43], the Zeeman splitting cannot be fully resolved. We believe that our results exhibit a more trustable initial slope, in view of the results arising from ChPT.

\section{SUMMARY AND CONCLUSIONS}

In this work, we have explored the effect of a strong external uniform magnetic field on diquark and nucleon masses. This has been done in the framework of a twoflavor Nambu-Jona-Lasinio effective model for low-energy QCD dynamics, including scalar quark-quark color pairing interactions to account for the diquarks. The relative strength of these interactions is determined by a coupling constant ratio $H / G$, where $H$ and $G$ are the coupling constants driving the scalar quark-quark and pseudoscalar quark-antiquark interactions, respectively. We have considered values of this ratio in the usually studied range $0.75 \leq H / G \leq 1.2$.

As done in the case of pions, diquarks have been treated as quantum fluctuations in the random phase approximation. Because of the presence of the external field, translational invariance turns out to be broken, as signaled by the presence of nonvanishing Schwinger phases, and the usual momentum basis cannot be used to diagonalize the corresponding polarization function. A proper basis can be found following the method introduced in Ref. [16] for charged pions, based on the Ritus eigenfunction approach to magnetized relativistic systems. In view of the nonrenormalizability of the NJL model, we have adopted as regularization procedure the magnetic-field-independent regularization scheme, as suggested from the scheme comparison performed in Ref. [49]. From the regularized diagonal polarization function, we have obtained the lowest Landau level diquark pole mass $m_{\Delta}$ and the magnetic-fielddependent mass $E_{\Delta}$, defined as the lowest quantummechanically allowed diquark energy. The numerical results for these quantities show that for low values of $|e B|$ the curves for both $m_{\Delta}$ and $E_{\Delta}$ lie below those corresponding to a pointlike diquark. This is reversed for $|e B|$ larger than $\sim 0.3-0.5 \mathrm{GeV}^{2}$, where the growth of $E_{\Delta}$ gets steeper in comparison with the pointlike case. It is also found that the increase of the magnetic-field-dependent mass becomes more pronounced for lower values of the ratio $H / G$.

Regarding the analysis of baryon states, in our framework nucleons have been built as bound quark-diquark states following a relativistic Fadeev approach in which only the formerly discussed scalar diquark channel is included. Given the complexity of the problem, we have 
considered a static approximation in which one disregards the momentum dependence of the exchanged quark. This approximation has been shown to lead to an adequate description of nucleon properties in the absence of external fields [54]. Once again, owing to the presence of nonvanishing Schwinger phases for charged particles, in the theoretical analysis we have made use of the Ritus eigenfunction method. In addition, we have introduced a further model parameter $\Lambda_{B}$ to regularize the otherwise divergent quark-diquark loops, for which we have chosen the proper time regularization scheme. We have found that for values of $H / G$ larger than 1 no value of $\Lambda_{B}$ is compatible with a physical value of the nucleon mass at zero external magnetic field.

We have obtained numerical results for the magnetic field dependence of the lowest-energy nucleon states, usually interpreted as the nucleon masses. In general, it is seen that the masses initially decrease for increasing magnetic field, whereas they show a steady growth for large values of $|e B|$. In the case of the proton, the results are found to depend strongly on the ratio $H / G$. It is also seen that the negative slopes of the mass curves at $B=0$ lead to the phenomenologically correct signs for the nucleon magnetic moments. Moreover, there is a qualitative agreement with ChPT results, although the slopes in our model are found to be somewhat lower. This conduces to numerical absolute values for the proton and neutron magnetic moments that are relatively small in comparison with the empirical ones.
The work presented in this article represents a first approach to relativistic magnetized nucleons as bound quark-diquark states within the NJL model. An improvement on the predictions for the nucleon magnetic moments is expected to be obtained by including axial vector diquark interactions. Moreover, a full calculation would require one to take into account the momentum dependence of the exchanged quark. We expect to report on these issues in future publications.

\section{ACKNOWLEDGMENTS}

We thank S. Noguera for useful discussions. This work has been supported in part by Consejo Nacional de Investigaciones Científicas y Técnicas and Agencia Nacional de Promoción Científica y Tecnológica (Argentina), under Grants No. PIP17-700 and No. PICT17-03-0571, respectively, and by the National University of La Plata (Argentina), Project No. X824.

\section{APPENDIX A: DIAGONALIZATION OF $\mathbb{D}_{\bar{P}_{\bar{P}}^{\prime}}^{(p)}$ IN RITUS SPACE}

In this Appendix, we briefly sketch how to prove that the Dirac operator $\mathbb{D}_{\bar{P} \bar{P}^{\prime}}^{(p)}$ in Eq. (60) is diagonal. Let us start by taking into account the integral $I_{\bar{P} \bar{P}^{\prime}}^{\lambda, \lambda^{\prime}}(q, r)$ in Eq. (62). Denoting $w=x_{1}-z_{1}$ and integrating over the remaining space variables, it is easy to show that

$$
I_{\bar{P} \bar{P}^{\prime}}^{\lambda, \lambda^{\prime}}(q, r)=(2 \pi)^{6} \delta^{(2)}\left(P_{\|}-P_{\|}^{\prime}\right) \delta\left(P_{2}-P_{2}^{\prime}\right) \delta^{(2)}\left(q_{\|}+r_{\|}-P_{\|}\right) G_{k_{\lambda}, k_{\lambda^{\prime}}^{\prime}}\left(q_{\perp}+r_{\perp}\right),
$$

where

$$
\begin{aligned}
G_{k_{\lambda}, k_{\lambda^{\prime}}^{\prime}}\left(q_{\perp}+r_{\perp}\right)= & \frac{(-1)^{k_{\lambda}+k_{\lambda^{\prime}}^{\prime}}}{B_{p}} \int_{0}^{\infty} d w e^{i\left(q_{1}+r_{1}\right) w} N_{k_{\lambda}} D_{k_{\lambda}}\left(s_{p} \sqrt{2 / B_{p}}\left(q_{2}+r_{2}\right)-\sqrt{B_{p} / 2} w\right) \\
& \times N_{k_{\lambda^{\prime}}^{\prime}} D_{k_{\lambda^{\prime}}^{\prime}}\left(s_{p} \sqrt{2 / B_{p}}\left(q_{2}+r_{2}\right)+\sqrt{B_{p} / 2} w\right) .
\end{aligned}
$$

The integral over $w$ can be carried out using the following property:

$$
\int_{0}^{\infty} d \psi e^{i \gamma \psi} D_{\ell}(\eta-\psi) D_{n}(\eta+\psi)= \begin{cases}(-1)^{\ell} \sqrt{2 \pi} \ell ! e^{-\frac{\gamma^{2}+\eta^{2}}{2}}(i \gamma+\eta)^{n-\ell} L_{\ell}^{n-\ell}\left(\eta^{2}+\gamma^{2}\right) & \text { if } n \geq \ell, \\ (-1)^{n} \sqrt{2 \pi} n ! e^{-\frac{\gamma^{2}+\eta^{2}}{2}}(-i \gamma+\eta)^{\ell-n} L_{n}^{\ell-n}\left(\eta^{2}+\gamma^{2}\right) & \text { if } \ell \geq n .\end{cases}
$$

Assuming that $k_{\lambda^{\prime}}^{\prime} \geq k_{\lambda}$ (the analysis is similar for the other case), one has

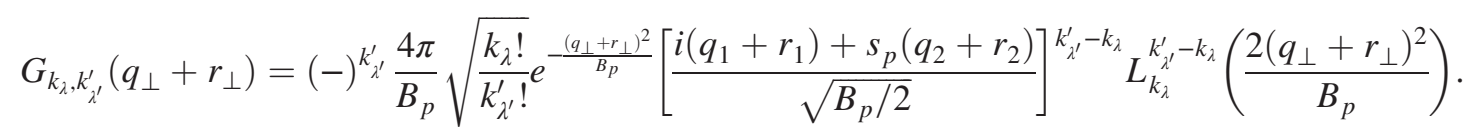


Now let us take this result to carry out the integral over perpendicular momenta in Eq. (60):

$$
I_{\perp}=\int_{q_{\perp} r_{\perp}} \sum_{\lambda, \lambda^{\prime}} G_{k_{\lambda}, k_{\lambda^{\prime}}^{\prime}}\left(q_{\perp}+r_{\perp}\right) \tilde{\mathcal{G}}_{\Delta}\left(q_{\perp}, q_{\|}\right) \Gamma_{\lambda} \tilde{S}^{u}\left(r_{\perp}, P_{\|}-q_{\|}\right) \Gamma_{\lambda^{\prime}}
$$

Using the form of the quark propagator in Eq. (15), it can be seen that the product $\Gamma_{\lambda} \tilde{S}^{u}\left(r_{\perp}, P_{\|}-q_{\|}\right) \Gamma_{\lambda^{\prime}}$ can be written as

$$
\Gamma_{\lambda} \tilde{S}^{u}\left(r_{\perp}, P_{\|}-q_{\|}\right) \Gamma_{\lambda^{\prime}}=\mathcal{A}\left(r_{\perp}, P_{\|}-q_{\|}\right) \delta_{\lambda \lambda^{\prime}} \Gamma_{\lambda}+\mathcal{B}\left(r_{\perp}, P_{\|}-q_{\|}\right) r_{\perp} \cdot \gamma_{\perp} \delta_{-\lambda \lambda^{\prime}} \Gamma_{-\lambda}
$$

where $\mathcal{A}\left(r_{\perp}, P_{\|}-q_{\|}\right)$and $\mathcal{B}\left(r_{\perp}, P_{\|}-q_{\|}\right)$are functions of $r_{\perp}^{2}$. Then we get

$$
\begin{aligned}
I_{\perp}= & \int_{q_{\perp} r_{\perp}} \tilde{\mathcal{G}}_{\Delta}\left(q_{\perp}, q_{\|}\right) \sum_{\lambda}\left[G_{k_{\lambda}, k_{\lambda}^{\prime}}\left(q_{\perp}+r_{\perp}\right) \mathcal{A}\left(r_{\perp}, P_{\|}-q_{\|}\right) \Gamma_{\lambda}\right. \\
& \left.+G_{k_{\lambda}, k_{-\lambda}^{\prime}}\left(q_{\perp}+r_{\perp}\right) \mathcal{B}\left(r_{\perp}, P_{\|}-q_{\|}\right)\left(r_{1}-i \lambda r_{2}\right) \gamma_{\lambda} \Gamma_{-\lambda}\right]
\end{aligned}
$$

where $\gamma_{\lambda}=\left(\gamma_{1}+i \lambda \gamma_{2}\right) / 2$. To carry out the angular integrals in Eq. (A7), it is convenient to use polar coordinates, namely, $\vec{q}_{\perp}=(\tilde{q} \cos \theta, \tilde{q} \sin \theta), \vec{r}_{\perp}=(\tilde{r} \cos \varphi, \tilde{r} \sin \varphi)$. Noticing that the diquark propagator depends only on the squared momenta $q_{\|}^{2}$ and $q_{\perp}^{2}$ [see Eq. (42)], from Eq. (A4), we get

$$
\begin{aligned}
I_{\perp}= & \int_{0}^{\infty} \frac{\tilde{q} d \tilde{q}}{(2 \pi)^{2}} \int_{0}^{\infty} \frac{\tilde{r} d \tilde{r}}{(2 \pi)^{2}} \tilde{\mathcal{G}}_{\Delta}\left(\tilde{q}, q_{\|}\right) \sum_{\lambda} \\
& \times\left[\mathcal{A}\left(\tilde{r}, P_{\|}-q_{\|}\right) \Gamma_{\lambda} \int_{0}^{2 \pi} d \varphi e^{-i s_{p}\left(k_{\lambda}^{\prime}-k_{\lambda}\right) \varphi} \int_{0}^{2 \pi} d \theta F_{k_{\lambda}, k_{\lambda}^{\prime}}(\tilde{q}, \tilde{r}, \theta-\varphi)\right. \\
& \left.+\tilde{r} \mathcal{B}\left(\tilde{r}, P_{\|}-q_{\|}\right) \gamma_{\lambda} \Gamma_{-\lambda} \int_{0}^{2 \pi} d \varphi e^{-i\left[s_{p}\left(k_{-\lambda}^{\prime}-k_{\lambda}\right)+\lambda\right] \varphi} \int_{0}^{2 \pi} d \theta F_{k_{\lambda}, k_{-\lambda}^{\prime}}(\tilde{q}, \tilde{r}, \theta-\varphi)\right],
\end{aligned}
$$

where $F_{k_{\lambda}, k_{\lambda^{\prime}}^{\prime}}$ is a function that depends on $\theta-\varphi$ only through periodic functions $\sin (\theta-\varphi)$ and $\cos (\theta-\varphi)$. Taking into account that

$k_{\lambda}^{\prime}-k_{\lambda}=k^{\prime}-k, \quad s_{p}\left(k_{-\lambda}^{\prime}-k_{\lambda}\right)+\lambda=s_{p}\left(k^{\prime}-k\right)$,

and using the periodicity of the function $F_{k_{\lambda}, k_{\lambda^{\prime}}^{\prime}}$, it is seen that $I_{\perp}$ is proportional to

$$
\int_{0}^{2 \pi} d \varphi e^{-i s_{p}\left(k^{\prime}-k\right)}=2 \pi \delta_{k k^{\prime}}
$$

Together with the result in Eq. (A1), this shows that $\mathbb{D}_{\bar{P} \bar{P}^{\prime}}^{(p)}$ is proportional to $\hat{\delta}_{\bar{P} \bar{P}^{\prime}}$.

\section{APPENDIX B: EXPANSION AROUND $B=0$}

In this Appendix, we provide some hints for the expansions of the coefficients $\hat{X}_{ \pm}^{(N)}$ and $\hat{Y}_{ \pm}^{(N)}$ in Eqs. (71) and (72) around $B=0$. These expansions allow us to obtain the expressions for $\hat{X}$ and $\hat{Y}$ in Eqs. (73) and (74), as well as the slopes $\alpha_{N}$ in Eqs. (81).
The coefficients $\hat{X}_{ \pm}^{(N)}$ and $\hat{Y}_{ \pm}^{(N)}$ depend on $B$ both explicitly and implicitly, through $\mathcal{M}_{N}$ and $M$. In fact, it can be seen that $d M /\left.d B\right|_{B=0}=0$; hence, the effective quark mass $M$ can be taken as a constant at the lowest order in an expansion in powers of $|B|$. In this way, from Eqs. (71) and (72), the slopes $d \mathcal{M}_{N} / d|B|$ at $B=0$ are given by

$$
\alpha_{N}=\frac{\left.\frac{\partial \hat{X}_{\lambda}^{(N)}}{\partial|B|}\right|_{B=0}-\left.m_{N} \frac{\partial \hat{Y}_{\lambda}^{(N)}}{\partial|B|}\right|_{B=0}}{\hat{Y}-\frac{\partial \hat{X}}{\partial m_{N}}+m_{N} \frac{\partial \hat{Y}}{\partial m_{N}}},
$$

where appropriate values of $\lambda$ should be taken for $N=p$ and $N=n$ (see the discussion in the main text).

In particular, the partial derivatives in the numerator of the rhs of Eq. (B1) have to be calculated with some care due to the sums over Landau levels in Eqs. (76)-(79). As an example, let us consider the expression for $\hat{X}_{s_{p}}^{(p)}$ in Eq. (76). The factors that depend explicitly on the magnetic field can be expanded as 


$$
\begin{aligned}
\frac{B_{u}\left(1+t_{u}\right)}{B_{u}+\left(B_{p}+B_{\Delta}\right) t_{u}} & =1+\frac{\tau}{\Lambda_{B}^{2}}\left(B_{u}-B_{p}-B_{\Delta}\right)+\mathcal{O}\left(B^{2}\right), \\
B_{\Delta}\left[\frac{B_{u}+\left(B_{p}-B_{\Delta}\right) t_{u}}{B_{u}+\left(B_{p}+B_{\Delta}\right) t_{u}}\right]^{\ell} & =B_{\Delta} e^{-2 \tau \ell B_{\Delta} / \Lambda_{B}^{2}}\left[1+\frac{2 \tau^{2} \ell B_{\Delta} B_{p}}{\Lambda_{B}^{4}}+\mathcal{O}\left(B^{2}, \ell B^{3}\right)\right], \\
\mathcal{G}_{\Delta}^{(\mathrm{reg})}\left(\ell, q_{\|}^{2}\right) & =\mathcal{G}_{\Delta, B=0}^{(\mathrm{reg})}\left(q_{\|}^{2}+2 \ell B_{\Delta}\right)+\left.\frac{d \mathcal{G}_{\Delta, B=0}^{(\mathrm{reg})}\left(q^{2}\right)}{d q^{2}}\right|_{q^{2}=q_{\|}^{2}+2 \ell B_{\delta}} B_{\Delta}+\mathcal{O}\left(B^{2}, \ell B^{3}\right) .
\end{aligned}
$$

For the evaluation of the sum over Landau levels in the limit of a low magnetic field, one can use the relation

$$
B \sum_{\ell=0}^{\infty} e^{-\alpha \ell B} F(\ell B)=\int_{0}^{\infty} d x e^{-\alpha x} F(x)+\frac{1}{2} F(0) B+\mathcal{O}\left(B^{2}\right),
$$

which is valid for $\alpha>0$ if the function $F(x)$ allows a Taylor expansion around $x=0$ and is well behaved at $x \rightarrow \infty$. In this way, after an integration by parts, one arrives at

$$
\begin{aligned}
& B_{\Delta} \frac{B_{u}\left(1+t_{u}\right)}{B_{u}+\left(B_{p}+B_{\Delta}\right)} \sum_{\ell=0}^{\infty}\left[\frac{B_{u}+\left(B_{p}-B_{\Delta}\right) t_{u}}{B_{u}+\left(B_{p}+B_{\Delta}\right) t_{u}}\right]^{\ell} \mathcal{G}_{\Delta}^{(\mathrm{reg})}\left(\ell, q_{\|}^{2}\right) \\
& \quad=\frac{1}{2} \int_{0}^{\infty} d \omega e^{-\tau \omega / \Lambda_{B}^{2}} \mathcal{G}_{\Delta, B=0}^{(\mathrm{reg})}\left(q_{\|}^{2}+\omega\right)\left[1+\frac{\tau}{\Lambda_{B}^{2}}\left(B_{u}-B_{p}\right)+\frac{\omega \tau^{2} B_{p}}{\Lambda_{B}^{4}}+\mathcal{O}\left(B^{2}\right)\right] .
\end{aligned}
$$

The variable $\omega$ can be identified with the perpendicular component of the momentum squared, $q_{\perp}^{2}$, in the $B \rightarrow 0$ limit. In addition, with the aid of some properties of the Bessel functions, one can prove the relations

$$
\begin{aligned}
\int_{0}^{\infty} d q_{\|}^{2} \int_{0}^{\infty} d q_{\perp}^{2} J_{0}\left(\alpha q_{\|}\right) f\left(q_{\|}^{2}+q_{\perp}^{2}\right) & =\frac{4}{\alpha} \int_{0}^{\infty} d q q^{2} J_{1}(\alpha q) f\left(q^{2}\right), \\
\int_{0}^{\infty} d q_{\|}^{2} \int_{0}^{\infty} d q_{\perp}^{2} q_{\perp}^{2} J_{0}\left(\alpha q_{\|}\right) f\left(q_{\|}^{2}+q_{\perp}^{2}\right) & =\frac{8}{\alpha^{2}} \int_{0}^{\infty} d q q^{3} J_{2}(\alpha q) f\left(q^{2}\right) .
\end{aligned}
$$

Now, using Eqs. (B4) and (B5), it is easy to see that

$$
\left.\hat{X}_{s_{p}}^{(p)}\right|_{B=0}=\hat{X},\left.\quad \frac{\partial \hat{X}_{s_{p}}^{(p)}}{\partial|B|}\right|_{B=0}=\left(Q_{p}-Q_{u}\right) \mathcal{I}_{1}-Q_{p} \mathcal{I}_{2},
$$

where $\hat{X}$ and $\mathcal{I}_{k}$ are given by Eqs. (73) and (83), respectively.

A similar procedure can be followed in order to obtain the expansions for $\hat{Y}_{s_{p}}^{(p)}, \hat{X}_{\lambda}^{(n)}$, and $\hat{Y}_{\lambda}^{(n)}$. The evaluation of the derivatives in the denominator of Eq. (B1) is straightforward, leading to the final expressions of $\alpha_{p}$ and $\alpha_{n}$ in Eqs. (81).

[1] D. E. Kharzeev, K. Landsteiner, A. Schmitt, and H. U. Yee, Lect. Notes Phys. 871, 1 (2013).

[2] J. O. Andersen, W. R. Naylor, and A. Tranberg, Rev. Mod. Phys. 88, 025001 (2016).

[3] V. A. Miransky and I. A. Shovkovy, Phys. Rep. 576, 1 (2015).

[4] D. Grasso and H. R. Rubinstein, Phys. Rep. 348, 163 (2001).

[5] D. E. Kharzeev, L. D. McLerran, and H. J. Warringa, Nucl. Phys. A803, 227 (2008); V. Skokov, A. Y. Illarionov, and
V. Toneev, Int. J. Mod. Phys. A 24, 5925 (2009); V. Voronyuk, V. Toneev, W. Cassing, E. Bratkovskaya, V. Konchakovski, and S. Voloshin, Phys. Rev. C 83, 054911 (2011); W. T. Deng and X. G. Huang, Phys. Rev. C 85, 044907 (2012).

[6] R. C. Duncan and C. Thompson, Astrophys. J. Lett. 392, L9 (1992); C. Kouveliotou et al., Nature (London) 393, 235 (1998).

[7] S. Fayazbakhsh, S. Sadeghian, and N. Sadooghi, Phys. Rev. D 86, 085042 (2012). 
[8] S. Fayazbakhsh and N. Sadooghi, Phys. Rev. D 88, 065030 (2013).

[9] S. S. Avancini, W. R. Tavares, and M. B. Pinto, Phys. Rev. D 93, 014010 (2016).

[10] S. S. Avancini, R. L. S. Farias, M. Benghi Pinto, W. R. Tavares, and V. S. Timoteo, Phys. Lett. B 767, 247 (2017).

[11] S. Mao and Y. Wang, Phys. Rev. D 96, 034004 (2017).

[12] R. Zhang, W. j. Fu, and Y. x. Liu, Eur. Phys. J. C 76, 307 (2016).

[13] D. G. Dumm, M. F. I. Villafañe, and N. N. Scoccola, Phys. Rev. D 97, 034025 (2018).

[14] Z. Wang and P. Zhuang, Phys. Rev. D 97, 034026 (2018).

[15] H. Liu, X. Wang, L. Yu, and M. Huang, Phys. Rev. D 97, 076008 (2018).

[16] M. Coppola, D. G. Dumm, and N. N. Scoccola, Phys. Lett. B 782, 155 (2018).

[17] M. Coppola, D. G. Dumm, S. Noguera, and N. N. Scoccola, Phys. Rev. D 100, 054014 (2019).

[18] S. Mao, Phys. Rev. D 99, 056005 (2019).

[19] S. S. Avancini, R. L. S. Farias, and W. R. Tavares, Phys. Rev. D 99, 056009 (2019).

[20] A. Ayala, R. L. S. Farias, S. Hernández-Ortiz, L. A. Hernández, D. M. Paret, and R. Zamora, Phys. Rev. D 98, 114008 (2018).

[21] K. Kamikado and T. Kanazawa, J. High Energy Phys. 03 (2014) 009.

[22] N. O. Agasian and I. A. Shushpanov, J. High Energy Phys. 10 (2001) 006.

[23] J. O. Andersen, J. High Energy Phys. 10 (2012) 005.

[24] G. Colucci, E. S. Fraga, and A. Sedrakian, Phys. Lett. B 728, 19 (2014).

[25] V. D. Orlovsky and Y. A. Simonov, J. High Energy Phys. 09 (2013) 136.

[26] M. A. Andreichikov, B. O. Kerbikov, E. V. Luschevskaya, Y. A. Simonov, and O. E. Solovjeva, J. High Energy Phys. 05 (2017) 007.

[27] Y. A. Simonov, Yad. Fiz. 79, 277 (2016) [Phys. At. Nucl. 79, 455 (2016)].

[28] M. A. Andreichikov and Y. A. Simonov, Eur. Phys. J. C 78, 902 (2018).

[29] C. A. Dominguez, M. Loewe, and C. Villavicencio, Phys. Rev. D 98, 034015 (2018).

[30] G. S. Bali, F. Bruckmann, G. Endrodi, Z. Fodor, S. D. Katz, S. Krieg, A. Schafer, and K. K. Szabo, J. High Energy Phys. 02 (2012) 044.

[31] Y. Hidaka and A. Yamamoto, Phys. Rev. D 87, 094502 (2013).
[32] E. V. Luschevskaya, O. E. Solovjeva, O. A. Kochetkov, and O. V. Teryaev, Nucl. Phys. B898, 627 (2015).

[33] B. B. Brandt, G. Bali, G. Endrodi, and B. Glaessle, Proc. Sci., LATTICE2015 (2016) 265 [arXiv:1510.03899].

[34] G. S. Bali, B. B. Brandt, G. Endrodi, and B. Glaessle, Phys. Rev. D 97, 034505 (2018).

[35] H. T. Ding, S. T. Li, A. Tomiya, X. D. Wang, and Y. Zhang, arXiv:2008.00493.

[36] B. C. Tiburzi, Nucl. Phys. A814, 74 (2008).

[37] A. Deshmukh and B. C. Tiburzi, Phys. Rev. D 97, 014006 (2018).

[38] H. Taya, Phys. Rev. D 92, 014038 (2015).

[39] A. Haber, F. Preis, and A. Schmitt, Phys. Rev. D 90, 125036 (2014).

[40] A. Mukherjee, S. Ghosh, M. Mandal, S. Sarkar, and P. Roy, Phys. Rev. D 98, 056024 (2018).

[41] B. R. He, Phys. Lett. B 765, 109 (2017).

[42] C. A. Dominguez, L. A. Hernandez, M. Loewe, C. Villavicencio, and R. Zamora, arXiv:2008.10742.

[43] G. Endrodi and G. Markó, J. High Energy Phys. 08 (2019) 036.

[44] U. Vogl and W. Weise, Prog. Part. Nucl. Phys. 27, 195 (1991).

[45] S. P. Klevansky, Rev. Mod. Phys. 64, 649 (1992).

[46] T. Hatsuda and T. Kunihiro, Phys. Rep. 247, 221 (1994).

[47] J. S. Schwinger, Phys. Rev. 82, 664 (1951).

[48] V. I. Ritus, Sov. Phys. JETP 48, 788 (1978).

[49] S. S. Avancini, R. L. S. Farias, N. N. Scoccola, and W. R. Tavares, Phys. Rev. D 99, 116002 (2019).

[50] R. T. Cahill, C. D. Roberts, and J. Praschifka, Aust. J. Phys. 42, 129 (1989).

[51] H. Reinhardt, Phys. Lett. B 244, 316 (1990).

[52] A. Buck, R. Alkofer, and H. Reinhardt, Phys. Lett. B 286, 29 (1992).

[53] S. z. Huang and J. Tjon, Phys. Rev. C 49, 1702 (1994).

[54] N. Ishii, W. Bentz, and K. Yazaki, Phys. Lett. B 301, 165 (1993).

[55] N. Ishii, W. Bentz, and K. Yazaki, Nucl. Phys. A587, 617 (1995).

[56] G. Hellstern and C. Weiss, Phys. Lett. B 351, 64 (1995).

[57] H. Asami, N. Ishii, W. Bentz, and K. Yazaki, Phys. Rev. C 51, 3388 (1995).

[58] H. Mineo, W. Bentz, N. Ishii, and K. Yazaki, Nucl. Phys. A703, 785 (2002).

[59] T. Primer, W. Kamleh, D. Leinweber, and M. Burkardt, Phys. Rev. D 89, 034508 (2014).

[60] M. Buballa, Phys. Rep. 407, 205 (2005). 